\title{
Enhanced Success History Adaptive DE for Parameter Optimization of Photovoltaic Models
}

\author{
Yingjie Song, ${ }^{1}$ Daqing Wu $\left(D,{ }^{2}\right.$ Ali Wagdy Mohamed $\left(D,{ }^{3,4}\right.$ Xiangbing Zhou, ${ }^{5}$ Bin Zhang, \\ and Wu Deng' \\ ${ }^{1}$ School of Computer Science and Technology, Shandong Technology and Business University, Yantai 264005, China \\ ${ }^{2}$ College of Economics \& Management, Shanghai Ocean University, Shanghai 201306, China \\ ${ }^{3}$ Operations Research Department, Institute of Statistical Studies and Research, Cairo University, Giza 12613, Egypt \\ ${ }^{4}$ Wireless Intelligent Networks Center (WINC), School of Engineering and Applied Sciences, Nile University, Giza, Egypt \\ ${ }^{5}$ School of Information and Engineering, Sichuan Tourism University, Chengdu 610100, China \\ ${ }^{6}$ College of Electronic Information and Automation, Civil Aviation University of China, Tianjin 300300, China
}

Correspondence should be addressed to Daqing Wu; dqwu@shou.edu.cn

Received 3 November 2020; Revised 18 November 2020; Accepted 28 November 2020; Published 29 January 2021

Academic Editor: Ahmed Mostafa Khalil

Copyright ( $) 2021$ Yingjie Song et al. This is an open access article distributed under the Creative Commons Attribution License, which permits unrestricted use, distribution, and reproduction in any medium, provided the original work is properly cited.

In the past few decades, a lot of optimization methods have been applied in estimating the parameter of photovoltaic (PV) models and obtained better results, but these methods still have some deficiencies, such as higher time complexity and poor stability. To tackle these problems, an enhanced success history adaptive DE with greedy mutation strategy (EBLSHADE) is employed to optimize parameters of PV models to propose a parameter optimization method in this paper. In the EBLSHADE, the linear population size reduction strategy is used to gradually reduce population to improve the search capabilities and balance the exploitation and exploration capabilities. The less and more greedy mutation strategy is used to enhance the exploitation capability and the exploration capability. Finally, a parameter optimization method based on EBLSHADE is proposed to optimize parameters of PV models. The different PV models are selected to prove the effectiveness of the proposed method. Comparison results demonstrate that the EBLSHADE is an effective and efficient method and the parameter optimization method is beneficial to design, control, and optimize the PV systems.

\section{Introduction}

In recent years, in order to solve the problems of environmental pollution and burn out, the utilization of solar, wind, hydropower, nuclear, and so on has been increasing attention [1]. Among them, solar energy is considered as one of the most promising alternatives to inexhaustible and clean sources. At the present time, the PV systems play a very important role in power system, because solar energy can directly be converted into electric energy to supply power with humans. Therefore, solar PV systems have been widely applied in the whole world and have continued growing. However, the PV systems are exposed to the external environment and their PV arrays are prone to aging, which seriously affects production efficiency of PV panels and are harmful to the work efficiency of solar energy [2, 3]. Hence, in order to effectively design, simulate, estimate, control, and optimize PV systems, it is paramount to estimate the performance using exact model in operation. The most widely used mathematical models for describing the nonlinear behaviour and performance are the single and double diode models and the PV model. However, the accurateness of these models is dependent on the parameters' values of models. The parameters are the reflection of the intrinsic characteristics of the PV model, and the I-V equation can be determined by identifying the PV parameters in order to predict the output power of PV array. The parameter optimization problem of the PV model is to fastly and accurately identify the parameters of the PV model in order to obtain better output power prediction and maximum power 
point tracking. Due to the aging, failure, breakdown, and unstable working conditions, it is very difficult to determine these parameters. Therefore, it is an extremely essential work to deeply study an effective method to optimize the parameters of PV and improve the solar energy utilizing efficiency.

Usually, the estimated problem of unknown parameters for the PV models is considered as an optimization objective function $[4,5]$. Due to the measured current data and voltage data involving noise, the constructed objective function is a nonlinear and multimodal function with multiple local optimums. Some researchers have studied and presented many methods to optimize the parameters of PV models in recent years, such as analytical method, deterministic method, and heuristic method. The heuristic method is a promising alternative to analytical method and deterministic method [6]. Due to no strict restrictions for the objective function, a lot of heuristic methods have obtained more and more attention for optimizing the parameters of PV models, such as simulated annealing (SA), genetic algorithm (GA), differential evolution (DE), particle swarm optimization (PSO), artificial bee colony (ABC), bacterial foraging algorithm (BFA), teaching-learning-based optimization (TLBO), whale optimization algorithm (COA), bird mating optimizer (BMO), month flame optimizer (MFO), and backtracking search algorithm (BSA) [7-20]. Recently, gaining-sharing knowledge-based algorithm has been proposed by Mohamed et al. [21]. Zagrouba et al. [22] used genetic algorithms to identify the parameters of PV solar cells and modules. Ishaque et al. [23] presented a penalty-based DE to extract the parameters of solar PV models. Merchaoui et al. [24] presented an improved PSO with adaptive mutation strategy to extract the parameters of PV solar cell/module. Yu et al. [25] presented a performance-guided JAYA algorithm to identify the parameters of PV cell and modules. Chen et al. [26] presented a teachinglearning-based ABC algorithm to estimate the parameters of the solar PV model. Li et al. [27] presented an improved teaching-learning-based optimization algorithm to extract the parameters of PV models. Oliva et al. [28] used ABC algorithm to identify the parameters of solar cells. Wu et al. [29] presented an improved ant lion optimizer to identify the parameters of the PV cell model. Ram et al. [30] presented a new hybrid bee pollinator flower pollination algorithm to estimate the parameters of solar PV. Oliva et al. [31] presented an improved chaotic WOA to estimate the parameters of PV cells. Xu and Wang [32] presented a hybrid flower pollination algorithm to estimate the parameters of PV modules. Chen et al. [33] presented a diversificationenhanced Harris Hawks optimizer to identify the parameters of PV modules. Long et al. [34] presented a new hybrid algorithm based on a grey wolf optimizer and cuckoo search to extract the parameters of solar PV models. Chen et al. [35] presented a novel opposition-based sine cosine approach with local search to estimate the parameters of PV models. Cai et al. [36] employed an orthogonal experiment method to optimize the parameters of the dust absorbing structure for PV panels. The results reported by these heuristic methods have achieved satisfied results, which indicate that these heuristic methods are promising alternative to identify the parameters of PV models. But because the parameter identification of PV models is a nonlinear and multimodal problem, their accuracy and reliability need to be further improved. In addition, many heuristic methods have their own parameters to be experimentally tuned to improve their efficacy, accuracy, reliability, and scalability.

Differential evolution (DE), a random evolution algorithm based on population evolution, was proposed by Storn and Price in 1997 [37], which has been regarded a simple and efficient optimization algorithm. Due to the fast convergence, robustness, and search ability, various advanced DE variants have been presented and widely applied in various fields of engineering design, operations research, biomedicine, and so on. Most of the various advanced DE variants are used to optimize the parameters of different PV models. Chang [38] presented an ant direction hybrid differential evolution algorithm. Gong and Cai [39] presented an improved adaptive differential evolution with crossover rate repairing technique and ranking-based mutation, as $\mathrm{R}-\mathrm{cr}$ IJADE. Muhsen et al. [40] presented an improved differential evolution with adaptive mutation per iteration algorithm (DEAM). Chellaswamy and Ramesh [41] presented adaptive differential evolution algorithm. Mohamed and Abdulaziz [42] proposed a differential evolution with novel mutation and adaptive crossover strategies to solve largescale global optimization problem. Zhang et al. [43] presented a gradient decent-based multiobjective cultural differential evolution (GD-MOCDE) to improve the optimal efficiency. Mohamed [44] proposed an enhanced adaptive differential evolution algorithm to solve large-scale global optimization problems. Rashidi and Khorshidi [45] presented a multiobjective differential evolution algorithm. Mohamed [44] presented a multiobjective self-adaptive differential evolution (MOSaDE) algorithm. Mohamed [46] proposed a new approach to differential evolution algorithm for solving stochastic programming problem. Ramli et al. [47] presented a multiobjective self-adaptive differential evolution algorithm. Mohamed and Suganthan [48] proposed enhanced fitness-adaptive differential evolution algorithm with novel mutation to solve real-parameter unconstrained optimization problem. Mohamed and Mohamed [49] proposed an adaptive guided directed differential evolution algorithm to solve unconstrained optimization problems. Tey et al. [50] presented an improved global search space differential evolution algorithm. Xiong et al. [51] presented an effective hybrid method based on the exploration of DE with the exploitation of WOA as DE/ WOA. Hadi et al. [52] proposed a LSHADE-SPA memetic framework to solve large-scale optimization problem. Mohamed and Mohamed [53] proposed an enhanced AGDE algorithm for real-parameter unconstrained optimization problem. Li et al. [54] presented a memetic adaptive differential evolution as MADE algorithm. Mohamed et al. [55] proposed an enhanced DE algorithm (EDDE) that utilizes the information given by good individuals and bad individuals in the population. The new mutation scheme maintains effectively the exploration/exploitation balance. Essiet et al. [56] presented an improved enhanced 
differential evolution algorithm for implementing demand response between the aggregator and consumer. In the team of professor Mohamed, a lot of work has been done to improve the DE algorithm. Mohamed et al. [53] proposed an enhanced directed differential evolution algorithm for solving constrained nonlinear integer and mixed-integer global optimization problems. Mohamed et al. [57] proposed an EBLSHADE algorithm based on novel mutation strategy. In this work, two mutation operators are introduced, ord_best and ord_pbest, which are versions of the classical DE/current-to-best/1 scheme. The proposed mutations were incorporated into SHADE and LSHADE algorithms in order to enhance their performances.

Due to the better robustness, stability, and quality of the solution of LSHADE, an enhanced SHADE (EBLSHADE) algorithm is applied to propose a parameter optimization method to optimize the parameters of PV models quickly, accurately, and reliably. In the EBLSHADE, a less and more greedy mutation strategy is used to enhance the exploitation capability and the exploration capability. A linear population size reduction strategy is used to gradually reduce population for improving the convergence speed, balancing the exploration and exploitation capabilities in the process of evolution. The EBLSHADE is employed to propose a parameter optimization method to optimize the unknown parameter estimation of the single diode model, double diode model, and PV model. The experimental results demonstrate that the parameter optimization method can exactly and reliably optimize the unknown parameters of different PV models and provide highly competitive results compared with other algorithms.

The main contributions of this paper are described as follows:

(i) An EBLSHADE is applied to effectively optimize the parameters of PV models. Based on quantified performance, the proper update strategy can adaptively be selected for individuals to immeasurably elevate the related searching performance.

(ii) The less and more greedy mutation strategy in the EBLSHADE is used to enhance the exploitation capability and the exploration capability, respectively.

(iii) The linear population size reduction strategy is employed to gradually reduce population to further improve the convergence speed, balance the exploration and exploitation capabilities, and avoid to falling into local optimum in the process of evolution.

(iv) The performance of EBLSHADE has been extensively investigated by the parameter estimation of different PV models.

The rest of this paper is arranged as follows. Section 2 describes different PV models and their objective functions. The differential evolution algorithm is briefly described in Section 3. Section 4 introduces an enhanced SHADE with multistrategies in detail. The experimental results on different PV models are shown and analyzed in Section 5. Finally, the conclusion is given in Section 6.

\section{Differential Evolution}

The main operations of DE contain initialization population, mutation operation, crossover operation, selection operation, and so on. Its main thoughts are to differentiate and scale between two different individual vectors in the same population and add a third individual vector in this population to obtain a mutation individual vector, which is crossed with the parent individual vector with a certain probability to generate an attempted individual vector. Finally, the attempted individual vector and the parent individual vector are executed greedy selection, and the better individual vector is saved to the next generation population. The basic evolution processes of $e \mathrm{DE}$ are described.

2.1. Abbreviations and Acronyms. The DE uses NP D-dimension vectors as the initial solution. Setting population number $\mathrm{N}$, each individual can be expressed as $x_{i}(G)=\left(x_{i 1}(G), x_{i 2}(G), \ldots, x_{i D}(G)\right)$. The initial population is generated in $\left[x_{\min }, x_{\max }\right]$.

$$
x_{i D}=x_{\min }+\operatorname{rand}(0,1) *\left(x_{\max }+x_{\min }\right),
$$

where $G$ represents the $G^{\text {th }}$ generation, $x_{\max }$ represents the maximum search space value, $x_{\min }$ represents the minimum search space value, and rand $(0,1)$ represents a random number.

2.2. Mutation Operation. It generates a mutation vector $V_{i, G}$ for $x_{i, G}$, namely, target vector. For each generated target vector, the mutation strategy is used to obtain a corresponding mutation vector. The mutation operation is the most important operation of DE. According to the different generation methods of the mutation individuals, several different mutation strategies of DE are formed. The most common mutation strategies are described as follows:

(1) DE/best/2/bin

$$
V_{i, G}=x_{\text {best }, G}+F \cdot\left(x_{r_{1}, G}-x_{r_{2}, G}\right)+F \cdot\left(x_{r_{3}, G}-x_{r_{4}, G}\right) \text {. }
$$

(2) $\mathrm{DE} / \mathrm{rand} / 2 / \mathrm{bin}$

$V_{i, G}=x_{r_{1}, G}+F \cdot\left(x_{r_{2}, G}-x_{r_{3}, G}\right)+F \cdot\left(x_{r_{4}, G}-x_{r_{5}, G}\right)$.

(3) $\mathrm{DE} /$ current-to-best/1/bin

$$
V_{i, G}=x_{i, G}+F \cdot\left(x_{\text {best }, G}-x_{i, G}\right)+F \cdot\left(x_{r_{1}, G}-x_{r_{2}, G}\right) \text {. }
$$

(4) $\mathrm{DE} /$ current-to-rand/1/bin

$$
V_{i, G}=x_{i, G}+F \cdot\left(x_{r 1, G}-x_{i, G}\right)+F \cdot\left(x_{r_{2}, G}-x_{r_{3}, G}\right) \text {. }
$$


2.3. Crossover Operation. Each pair of target vectors $x_{i, G}$ and the corresponding mutation vectors $V_{i, G}$ are crossed to obtain a test vector $U_{i, G}=\left(u_{1, G}, u_{2, G}, \ldots, u_{i, G}\right)$. In the basic $\mathrm{DE}$, it uses a binomial crossover to define.

$$
u_{i, G}= \begin{cases}v_{i, G}, & \text { if }\left(\operatorname{rand}_{j}(0,1) \leq C R\right) \text { or }\left(j=j_{\text {rand }}\right), \\ x_{i, G}, & \text { otherwise, }\end{cases}
$$

where the CR is a constant between 0 and 1 , which is used to control the duplicated proportion from the mutation vector. $j_{\text {rand }}$ is a selected integer within $[1, D], j=1,2,3, \ldots, D$.

2.4. Selection Operation. Comparing the objective function value $f\left(U_{i, G}\right)$ of each test vector, the objective function value of the test vector is less than the corresponding target vector, and then the target vector is replaced by the experimental vector. The selection operation is given as follows:

$$
X_{i, G+1}= \begin{cases}U_{i, G}, & \text { if }\left(f\left(U_{i, G}\right) \leq f\left(X_{i, G}\right)\right), \\ X_{i, G}, & \text { otherwise. }\end{cases}
$$

2.5. Implementation of DE. The DE algorithm evolves generation by generation until the result or ending condition has been met. The flow chart of DE algorithm is shown in Figure 1.

\section{EBLSHADE}

3.1. SHADE. The SHADE is one of the most successful variants of DE [57]. In the SHADE, a historical memory with $H$ entries is used. It is made up of MCR and MF, which can adaptively control parameters $\mathrm{CR}$ and $F$. In each iteration, each individual $\left(x_{i}\right)$ has its own $F_{i}$ and $\mathrm{CR}_{i}$ to generate a new test vector $u_{i}$. The two parameters are expressed as follows:

$$
\begin{aligned}
F_{i} & =\operatorname{rand} c_{i}\left(M_{F, r_{i}}, 0.1\right), \\
\mathrm{CR}_{i} & =\operatorname{rand} n_{i}\left(M_{C R, r_{i}}, 0.1\right),
\end{aligned}
$$

where $r_{i}$ is a random integer on $[1, H]$, rand $n$ is the Gaussian normal distribution, and the scale parameter is 0.1 , and rand $c$ is the Cauchy distribution and the variance is 0.1 .

The CR and $F$ of the generated test vectors are recorded as $S_{C R}$ and $S_{F}$. Their average values are stored in $M_{C R}$ and $M_{F}$. The SHADE keeps $H$ parameters to guide the control parameters in order to achieve self-adaptive search. Even if the $S_{C R}$ and $S_{F}$ of some offsprings contain a set of poor values, the stored parameters of the previous generation will not be affected. The control parameters are shown in Table 1.

Then, the $M_{\mathrm{CR}}$ and $M_{F}$ are updated according to the following expressions:

$$
\begin{aligned}
M_{\mathrm{CR}, \mathrm{t}+1} & = \begin{cases}\operatorname{Mean}_{\mathrm{WA}}\left(S_{\mathrm{CR}}\right), & \text { if } S_{\mathrm{CR}} \neq \varphi, \\
M_{\mathrm{CR}, \mathrm{t}}, & \text { otherwise, }\end{cases} \\
M_{\mathrm{F}, \mathrm{t}+1} & = \begin{cases}\operatorname{Mean}_{\mathrm{WL}}\left(S_{F}\right) & \text { if } S_{F} \neq \varphi, \\
M_{\mathrm{F}, \mathrm{t}}, & \text { otherwise, }\end{cases}
\end{aligned}
$$

where $t$ is an index to determine the saved position and $k$ is the set 1 . When a pair new $S_{C R}$ and $S_{F}$ are added in the history, $k$ is incremented by 1 . Mean ${ }_{\mathrm{WA}}\left(S_{\mathrm{CR}}\right)$ and Mean $\mathrm{WL}_{\mathrm{W}}$ $\left(S_{F}\right)$ are weight mean values of $S_{\mathrm{CR}}$ and weight Lehmer mean value of $S_{F}$.

3.2. LSHADE. An improved SHADE based on the reduction strategy of the linear population size, namely, LSHADE is developed. In the LSHADE, the population size is gradually lessened. Therefore, the linear function is described as follows:

$$
\mathrm{NP}_{G+1}=\operatorname{round}\left(\frac{\mathrm{NP}_{\min }-\mathrm{NP}_{0}}{\mathrm{Max}_{\mathrm{NFE}}} * \mathrm{NFE}+\mathrm{NP}_{0}\right) \text {, }
$$

where round returns the nearest integer number. NFE is the fitness optimization, $\operatorname{Max}_{\mathrm{NFE}}$ is the optimization with the maximum iterations, $\mathrm{NP}_{0}$ is the initial population size, and $\mathrm{NP}_{\text {min }}$ is the possible minimum population size, which is the minimum number of individuals $\left(\mathrm{NP}_{\min }=4\right)$.

\subsection{EBLSHADE}

3.3.1. A Mutation Strategy. The DE/current-to-best/1 strategy can find the best solution in the evolution. But it may deteriorate or lose the population diversity and exploration capability. To overcome these shortcomings, a variant of $\mathrm{DE} /$ current-to-best/1, namely, $\mathrm{DE} /$ current-toor_best/1 is used to balance the local exploitation and the global exploration abilities and enhance the convergence speed. In the strategy, all individuals are sorted to divide into three vectors. The best vector is referred as $x_{\text {or_best }}, G$, the median vector is referred as $x_{o r_{-} \text {median, }} G$, and the worst vector is referred as $x_{\text {or_worst }}, G$. Therefore, the trial vector is described as follows:

$$
\begin{aligned}
v_{i, G}= & x_{i, G}+F_{i} \cdot\left(x_{\text {or_best }, G}-x_{i, G}\right) \\
& +F_{i} \cdot\left(x_{\text {or_median, },}-x_{\text {or_worst }, G}\right) .
\end{aligned}
$$

As can be seen from the new mutation strategy equation (12), the added objective function value has two advantages. The difference vector of the best vector and the target vector is the first perturbation part of the new mutation strategy, which can substantially avoid prematurity and accelerate convergence. The second perturbation part of the new mutation strategy is the difference vector of the median vector and the worst vector. Therefore, the DE/current-toor_best/1 can get the global optimal solution.

When the population size reduction strategy can improve optimization performance of the algorithm, the initial population size will be reduced to 18 dimensions in the LSHADE. The increased population size will affect DE/ current-to-or_best/1; the probability of $x_{\text {or_best }}, G$, will be decreased to be the global best solution. Therefore, the behaviour of the DE/current-to-or_best/1 will approximate to $\mathrm{DE} / \mathrm{rand} / 1$. To solve this problem, an enhanced version of $\mathrm{DE} /$ current-to-or_pbest/1 is used. In this mutation strategy, one vector from top $p$ best vectors is included. The other two 


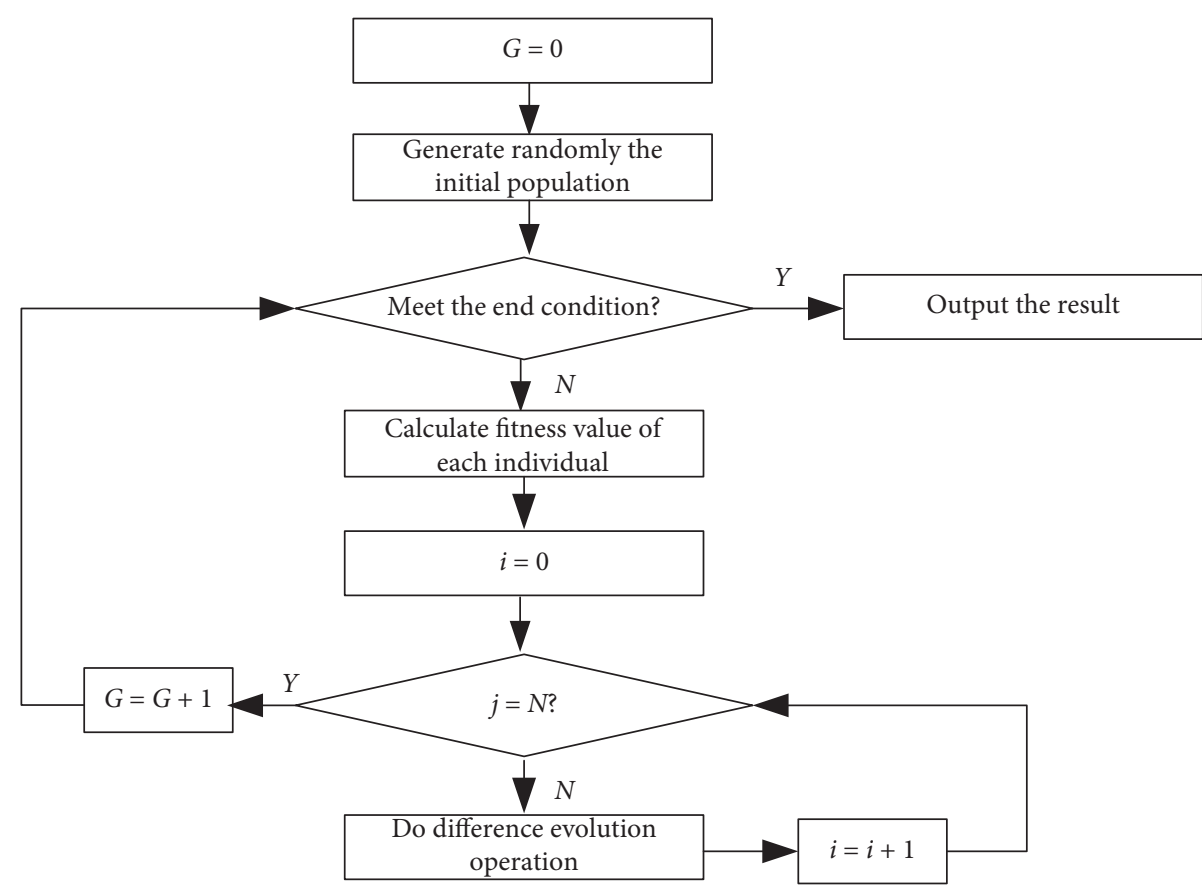

Figure 1: The flow of DE algorithm.

vectors are chosen randomly. Then, three vectors are sorted. The best vector is referred as $x_{\text {or_pbest }} G$, the median vector is referred as $x_{\text {or__pmedian }}, G$, and the worst vector is referred as $x_{\text {or_pworst }}, G$. Therefore, the trial vector is obtained as follows:

$$
\begin{aligned}
v_{\mathrm{i}, \mathrm{G}}= & x_{\mathrm{i}, \mathrm{G}}+F_{i} \cdot\left(x_{\text {or_pbest }, \mathrm{G}}-x_{\mathrm{i}, \mathrm{G}}\right) \\
& +F_{i} \cdot\left(x_{\text {or_pmedian }, \mathrm{G}}-x_{\text {or_pworst }, \mathrm{G}}\right) .
\end{aligned}
$$

3.3.2. Model of EBLSHADE. The flow of the EBLSHADE is shown in Figure 2.

\section{Modeling of PV Models}

4.1. SDM. This model can describe the behaviour of solar cell effectively [17]. Equivalent circuit is given in Figure 3.

In this model, it is made up of a leakage current shunt resistance, a parallel current source with a diode, and several resistors for the related load current loss. Therefore, the output current is described as follows:

$$
\begin{aligned}
I & =I_{\mathrm{ph}}-I_{d}-I_{\mathrm{sh}}, \\
I & =I_{\mathrm{ph}}-I_{d}\left[\exp \left(\frac{V+\mathrm{IR}_{s}}{\alpha V_{t}}\right)-1\right]-\frac{V+\mathrm{IR}_{s}}{R_{\mathrm{sh}}}, \\
V_{t} & =\frac{k \cdot T}{q} .
\end{aligned}
$$

From the abovementioned equations, the parameters of $I_{\mathrm{ph}}, I_{d}, R_{s}, R_{\mathrm{sh}}$, and $\alpha$ need to be optimized. Therefore, the objective functions can be formulated:

$$
\left\{\begin{array}{l}
f(V, I, x)=I_{\mathrm{ph}}-I_{d}\left[\exp \left(\frac{V+\mathrm{IR}_{s}}{\alpha V_{t}}\right)-1\right]-\frac{V+\mathrm{IR}_{s}}{R_{\mathrm{sh}}}-I, \\
x=\left\{I_{\mathrm{ph}}, I_{d}, R_{s}, R_{\mathrm{sh}}, \alpha\right\} .
\end{array}\right.
$$

4.2. DDM. It is used to take the recombination current loss effect. Equivalent circuit is given in Figure 4.

The output current of this model is described as follows:

$$
\begin{aligned}
I= & I_{\mathrm{ph}}-I_{d 1}-I_{d 2}-I_{\mathrm{sh}}, \\
I= & I_{\mathrm{ph}}-I_{\mathrm{sd} 1}\left[\exp \left(\frac{V+\mathrm{IR}_{s}}{\alpha_{1} V_{t}}\right)-1\right] \\
& -I_{\mathrm{sd} 2}\left[\exp \left(\frac{V+\mathrm{IR}_{s}}{\alpha_{2} V_{t}}\right)-1\right]-\frac{V+\mathrm{IR}_{s}}{R_{\mathrm{sh}}} .
\end{aligned}
$$

From the abovementioned equations, the parameters of $I_{\mathrm{ph}}, I_{\mathrm{sd} 1}, I_{\mathrm{sd} 2}, R_{s}, R_{\mathrm{sh}}, \alpha_{1}$, and $\alpha_{2}$ need to be estimated. Therefore, the objective functions can be formulated: 
TABLE 1: The control parameters.

\begin{tabular}{lcccc}
\hline Control parameter & 1 & Index & $H-1$ & $H$ \\
\hline$M_{\mathrm{CR}}$ & $M_{\mathrm{CR}, 1}$ & 2 & - & $M_{\mathrm{CR}, \mathrm{H}-1}$ \\
$M_{F}$ & $M_{\mathrm{F}, 1}$ & $M_{\mathrm{F}, \mathrm{H}-1}$ & $M_{\mathrm{CR}, \mathrm{H}}$ \\
\hline & $\left\{\begin{array}{l}f(V, I, x)=I_{\mathrm{ph}}-I_{\mathrm{sd} 1}\left[\exp \left(\frac{V+\mathrm{IR}_{s}}{\alpha_{1} V_{t}}\right)-1\right]-I_{\mathrm{sd} 2}\left[\exp \left(\frac{V+\mathrm{IR}_{s}}{\alpha_{2} V_{t}}\right)-1\right]-\frac{V+\mathrm{IR}_{s}}{R_{\mathrm{sh}}-I,} \\
\\
x=\left\{I_{\mathrm{ph}}, I_{\mathrm{sd} 1}, I_{\mathrm{sd} 2}, R_{s}, R_{\mathrm{sh}}, \alpha_{1}, \alpha_{2}\right\} .\end{array}\right.$ \\
\end{tabular}

4.3. PVM. It is usually based on series or parallel solar cells. Its equivalent circuit is described in Figure 5. This circuit can be extended to the $N$-diode PV model.

The output current of this model is formulated.

$$
I=I_{\mathrm{ph}} N_{p}-I_{d}\left[\exp \left(\frac{V+\left(\mathrm{IR}_{s} N_{s} / N_{p}\right)}{\alpha N_{s} V_{t}}\right)-1\right]-\frac{V+\left(R_{s} N_{s} / N_{p}\right)}{\left(R_{s} N_{s} / N_{p}\right)} .
$$

Due to the used PV model with series in the experiment, there is $N_{P}=1$. The abovementioned equation can be reformulated:

$$
I=I_{\mathrm{ph}}-I_{d}\left[\exp \left(\frac{V+\mathrm{IR}_{s} N_{s}}{\alpha N_{s} V_{t}}\right)-1\right]-\frac{V+R_{s} N_{s}}{R_{s h} N_{s}} .
$$

From the abovementioned equations, the parameters of $I_{\mathrm{ph}}, I_{d}, R_{s}, R_{\mathrm{sh}}$, and $\alpha$ will be optimized. The objective functions of the PV model can be formulated:

$$
\left\{\begin{array}{l}
f(V, I, x)=I_{\mathrm{ph}}-I_{d}\left[\exp \left(\frac{V+\mathrm{IR}_{s} N_{s}}{\alpha N_{s} V_{t}}\right)-1\right]-\frac{V+\mathrm{IR}_{s} N_{s}}{R_{\mathrm{sh}} N_{s}}-I, \\
x=\left\{I_{\mathrm{ph}}, I_{d}, R_{s}, R_{\mathrm{sh}}, \alpha\right\} .
\end{array}\right.
$$

\section{Experiment Results and Analysis}

5.1. Data and Environment. The benchmark experimental current-voltage data of $\operatorname{SDM}(\mathrm{Ns}=\mathrm{Np}=1)$ and $\mathrm{DDM}$ $(\mathrm{Ns}=1, \mathrm{~Np}=2)$ are used from reference [58]. The currentvoltage data came from references $[59,60]$. The experimental platform is a PC with an Intel i7-7700HQ, $16 \mathrm{~GB}$, under Windows 10 .

5.2. Parameter Setting. In order to guarantee the comparative fairness, the lower bound (LB) and upper bound (UP) are described in Table 2.

To prove the superior ability of the EBLSHADE, some existing algorithms are selected in Table 3. The parameters of the EBLSHADE are set as follows: $\mathrm{NP}=40, H=100$, $M_{\mathrm{CR}}=0.5^{*}$ ones $(1, \mathrm{H}), M_{F}=0.5^{*}$ ones $(1, \mathrm{H}), w 1=0.2$, $w 2=0.6, \mathrm{pmin}=0.05$, and $\mathrm{pmax}=0.2$. The settings of parameters are the same as the corresponding literatures for all compared algorithms. These algorithms are executed in Matlab2018b and 30 runs.

5.3. Root Mean Square Error. Root mean square error (RMSE) is the ratio square root of the square of the deviation between the predicted value and the actual value and the number of observations $n$. In the actual measurement, the observation number $n$ is always limited, and the true value can only be replaced by the most reliable value. It is used to measure the deviation between the observed value and the true value and illustrates the dispersion degree of the data. The root mean square error (RMSE) is defined as follows:

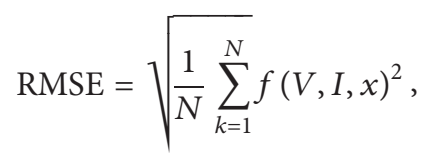

where $N$ is the experiment number and $x$ is a vector.

\subsection{Experiment Results}

5.4.1. Experimental Results of SDM. Here, the RMSE is the obtained computation value according to the equation of root mean square error (equation 20) by each independent run of the algorithm. MRMSE is the mean value of RMSE of different set of 30 runs; BRMSE is the best value of RMSE among different set of 30 runs. Therefore, the compared results of five parameters, statistical results of the best RMSE (BRMSE), the mean RMSE (MRMSE), the standard deviation (SD), and the least computing resources (NFE) are given in Table 3, where the obtained best results are bold. The boxplot of RMSE is presented in Figure 6 .

From Table 3 and Figure 6, the TLABC, MLBSA, JADE, SHADE, MADE, and EBLSHADE obtain the BRMSE value (9.8602E-04). Especially for MLBSA, SHADE, MADE, and EBLSHADE, the best, worst, and mean RMSE values are the same value $(\mathbf{9 . 8 6 0 2 E}-\mathbf{0 4})$. Due to the unavailable information, the RMSE is usually used to express the more accurate parameters. Although the second BRMSE value $(9.8603 \mathrm{E}-04)$ of IJAYA is infinitely close to the BRMSE value $(9.8602 \mathrm{E}-04)$, it is significant to reduce the order of objective function and improve the true value of parameters. For standard deviation, the EBLSHADE obtains the third best standard deviation value, which is close to the best 


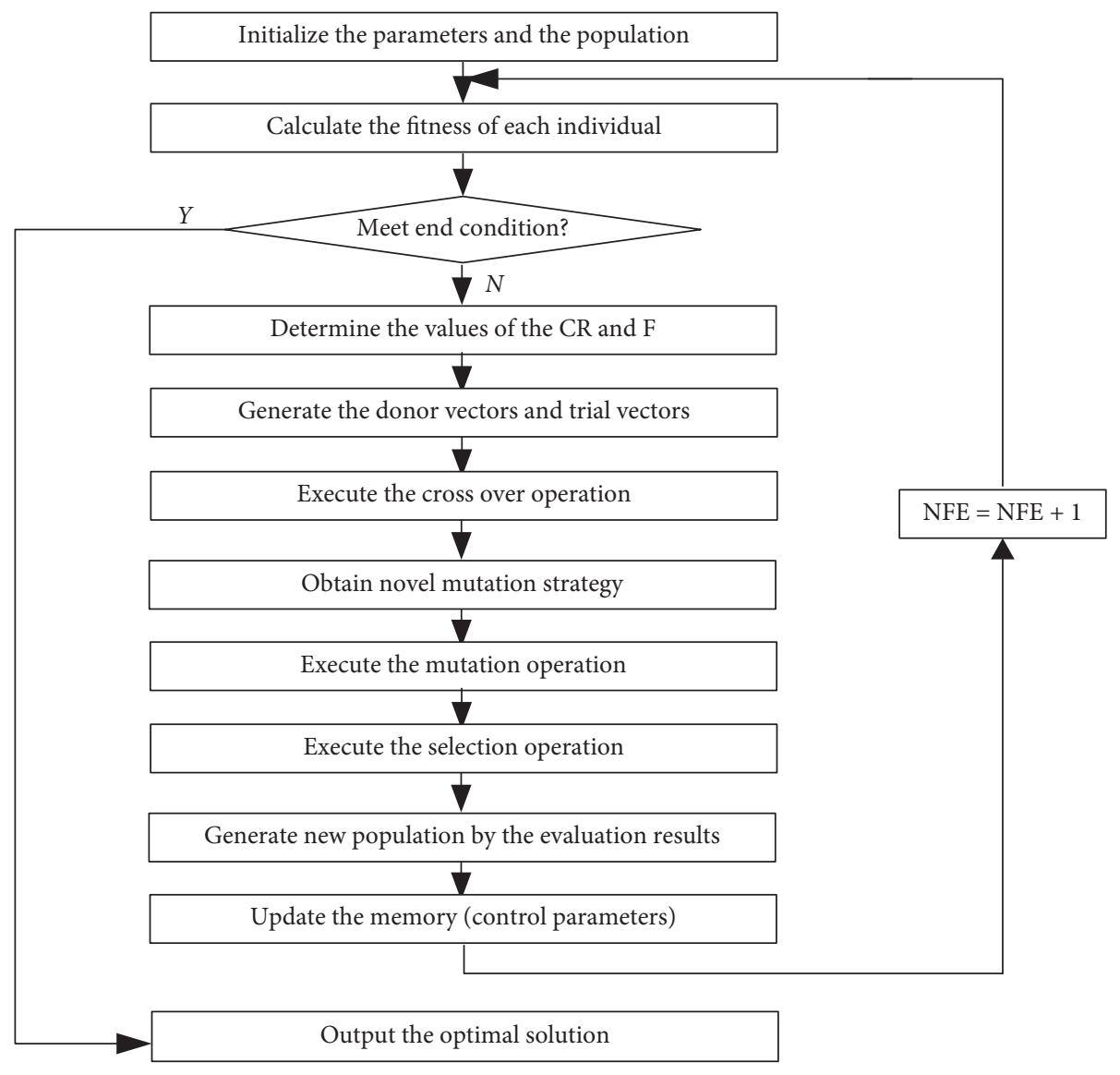

Figure 2: The flow of EBLSHADE.

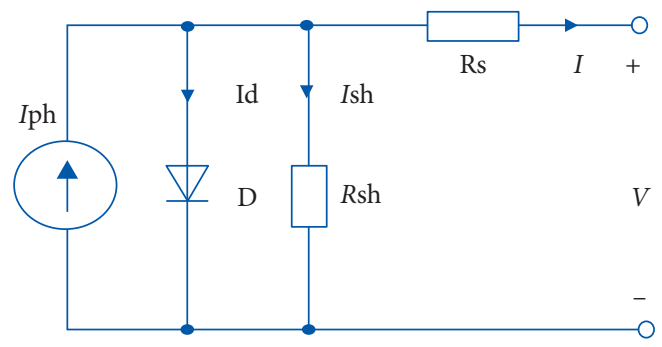

Figure 3: The equivalent circuit.

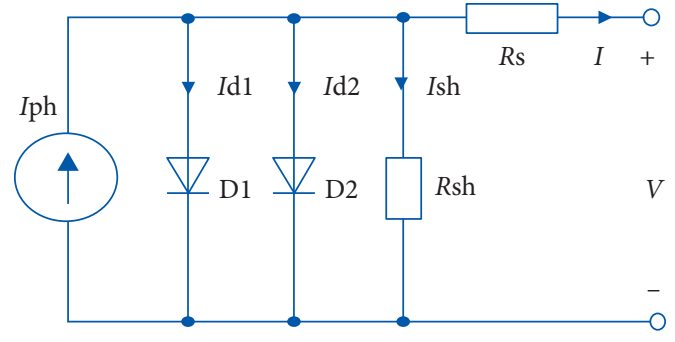

Figure 4: The equivalent circuits.

standard deviation value. For max NFE, the EBLSHADE required max NFE $=4,000$, which is much less than the other compared algorithms.
To further prove the effectiveness of the EBLSHADE, the detailed results of the individual absolute error of current (IAEI) and power (IAEP) between the experimental data and the measured data are shown in Table 4. See references for measurement data. The I-V and P-V characteristics curve obtained by EBLSHADE are shown in Figures 7-10. Note that Ic is calculated by the optimized parameters using EBLSHADE.

From Table 4 and Figures 7-10, all IAEI values are lesser than 2.5075E-03 and all IAEP values are lesser than $1.4626 \mathrm{E}-03$. It can be seen that the EBLSHADE can accurately optimize these parameters. It is also evident that the obtained experimental data by EBLSHADE are highly consistent with the measured data, which effectively reflects the optimized parameters to be accurate enough. Therefore, 


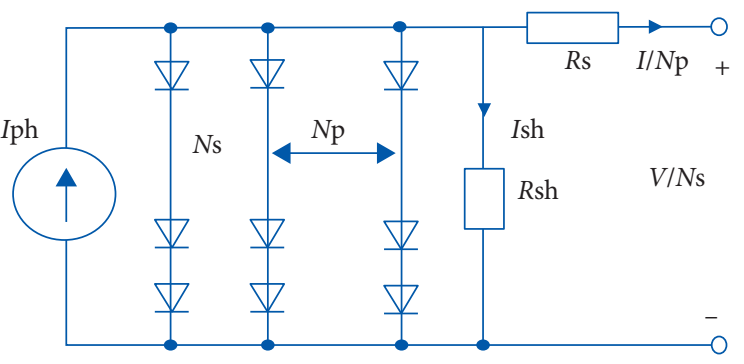

(a)

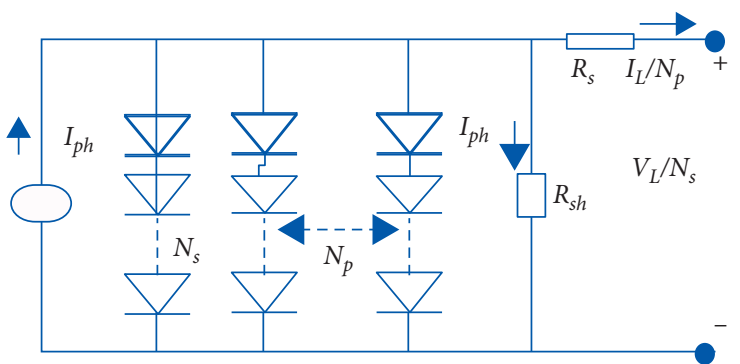

(b)

Figure 5: The equivalent circuit.

TABLE 2: Units for magnetic properties.

\begin{tabular}{|c|c|c|c|c|c|c|c|c|}
\hline \multirow[t]{2}{*}{ Parameters } & \multicolumn{2}{|c|}{$\begin{array}{l}\text { Single and double } \\
\text { diode models }\end{array}$} & \multicolumn{2}{|c|}{$\begin{array}{l}\text { Photowatt- } \\
\text { PWP201 }\end{array}$} & \multicolumn{2}{|c|}{ STP6-120/36 } & \multicolumn{2}{|c|}{ STM6-40/36 } \\
\hline & LB & UP & LB & UP & LB & UP & LB & UP \\
\hline$I_{\mathrm{ph}}(\mathrm{A})$ & 0 & 1 & 0 & 2 & 0 & 2 & 0 & 8 \\
\hline$I_{d}, I_{\mathrm{sd} 1}, I_{\mathrm{sd} 2}(\mu \mathrm{A})$ & 0 & 1 & 0 & 50 & 0 & 50 & 0 & 50 \\
\hline$R_{s}(\Omega)$ & 0 & 0.5 & 0 & 2 & 0 & 0.36 & 0 & 0.36 \\
\hline$R_{\mathrm{sh}}(\Omega)$ & 0 & 100 & 0 & 2000 & 0 & 1000 & 0 & 1500 \\
\hline$\alpha, \alpha_{1}, \alpha_{2}$ & 1 & 2 & 1 & 50 & 1 & 60 & 1 & 50 \\
\hline
\end{tabular}

Table 3: Comparison results.

\begin{tabular}{lccccccccc}
\hline Algorithm & $I_{\mathrm{ph}}(\mathrm{A})$ & $I_{d}(\mu \mathrm{A})$ & $R_{s}(\Omega)$ & $R_{\mathrm{sh}}(\Omega)$ & $\alpha$ & BRMSE & MRMSE & SD & $\mathrm{NFE}$ \\
\hline JAYA [61] & 0.7608 & 0.3281 & 0.0364 & 54.9298 & 1.4828 & $9.8946 \mathrm{E}-04$ & $1.1617 \mathrm{E}-03$ & $1.8796 \mathrm{E}-04$ & 50000 \\
IJAYA [61] & 0.7608 & 0.3228 & 0.0364 & 53.7595 & 1.4811 & $9.8603 \mathrm{E}-04$ & $9.9204 \mathrm{E}-04$ & $1.4033 \mathrm{E}-05$ & 50000 \\
GOTLBO [62] & 0.7608 & 0.3297 & 0.0363 & 53.3664 & 1.4833 & $9.8856 \mathrm{E}-04$ & $1.0450 \mathrm{E}-03$ & $5.0218 \mathrm{E}-05$ & 10000 \\
LETLBO [63] & 0.7608 & 0.3260 & 0.0363 & 53.7429 & 1.4821 & $9.8738 \mathrm{E}-04$ & $1.0333 \mathrm{E}-03$ & $4.6946 \mathrm{E}-05$ & 50000 \\
SATLBO [64] & 0.7608 & 0.3232 & 0.0364 & 53.7256 & 1.4812 & $9.8604 \mathrm{E}-04$ & $9.8780 \mathrm{E}-04$ & $2.300 \mathrm{E}-06$ & 50000 \\
DE/BBO [65] & 0.7605 & 0.3248 & 0.0364 & 55.2627 & 1.4817 & $9.9922 \mathrm{E}-04$ & $1.2948 \mathrm{E}-03$ & $2.5074 \mathrm{E}-04$ & 50000 \\
LBSA [66] & 0.7609 & 0.3258 & 0.0364 & 54.1083 & 1.4820 & $9.9125 \mathrm{E}-04$ & $1.1466 \mathrm{E}-03$ & $1.3482 \mathrm{E}-04$ & 50000 \\
CLPSO [62] & 0.7608 & 0.3430 & 0.0361 & 54.1965 & 1.4873 & $9.9633 \mathrm{E}-04$ & $1.0581 \mathrm{E}-03$ & $7.4854 \mathrm{E}-05$ & 50000 \\
BLSPO [67] & 0.7607 & 0.3662 & 0.0359 & 60.2845 & 1.4939 & $1.0272 \mathrm{E}-03$ & $1.3139 \mathrm{E}-03$ & $2.1166 \mathrm{E}-04$ & 50000 \\
CMM-DE/BBO [68] & 0.7608 & 0.3238 & 0.0364 & 53.8753 & 1.4814 & $9.8605 \mathrm{E}-04$ & $1.0486 \mathrm{E}-03$ & $8.1679 \mathrm{E}-03$ & 50000 \\
MLBSA [69] & 0.7608 & 0.0323 & 0.0364 & 53.7164 & 1.4812 & $\mathbf{9 . 8 6 0 2 E}-\mathbf{0 4}$ & $9.9852 \mathrm{E}-04$ & $1.8600 \mathrm{E}-05$ & 50000 \\
TLABC [26] & 0.7608 & 0.3316 & 0.0363 & 54.1154 & 1.4838 & $\mathbf{9 . 8 6 0 2 E}-\mathbf{0 4}$ & $\mathbf{9 . 8 6 0 2 E}-\mathbf{0 4}$ & $9.1500 \mathrm{E}-12$ & 50000 \\
JADE [58] & 0.7608 & 0.3229 & 0.0364 & 53.6477 & 1.4811 & $\mathbf{9 . 8 6 0 6 E}-\mathbf{0 4}$ & $1.0833 \mathrm{E}-03$ & $1.0900 \mathrm{E}-04$ & 10000 \\
SHADE [57] & 0.7608 & 0.3230 & 0.0364 & 53.7185 & 1.4812 & $\mathbf{9 . 8 6 0 2 E}-\mathbf{0 4}$ & $\mathbf{9 . 8 6 0 2 E}-\mathbf{0 4}$ & $2.2200 \mathrm{E}-11$ & 10000 \\
MADE [47] & 0.7608 & 0.3230 & 0.0364 & 53.7185 & 1.4812 & $\mathbf{9 . 8 6 0 2 E}-\mathbf{0 4}$ & $\mathbf{9 . 8 6 0 2 E}-\mathbf{0 4}$ & $2.7400 \mathrm{E}-15$ & 5000 \\
EBLSHADE & 0.7608 & 0.3230 & 0.0364 & 53.7185 & 1.4812 & $\mathbf{9 . 8 6 0 2 E}-\mathbf{0 4}$ & $\mathbf{9 . 8 6 0 2 E}-\mathbf{0 4}$ & $\mathbf{1 . 9 1 6 9 E}-\mathbf{1 5}$ & $\mathbf{4 0 0 0}$ \\
\hline
\end{tabular}

the EBLSHADE can be considered as a significant method for the parameter optimization of SDM.

5.4.2. Experimental Results of DDM. Seven optimized parameters will increase the optimization difficulty. The EBLSHADE is compared with the selected methods in Table 5. The results are shown in Table 6. The boxplot of RMSE is shown in Figure 11.

From Table 5 and Figure 11, the SHADE only achieved the BRMSE value $(\mathbf{9 . 8 2 4 8 E}-\mathbf{0 4})$, trailed by SATLBO, IJAYA, MLBSA, MADE, and EBLSHADE. The obtained BRMSE value $(9.8295 \mathrm{E}-04)$ by EBLSHADE is close to the obtained BRMSE value $(9.8248 E-04)$. By comparison, the
RMSE values of the other algorithm are lower, especially the obtained result by BLSPO is worst. While the SHADE consumed $20000 \mathrm{NFE}$, although the EBLSHADE did no't obtain the BRMSE value, it only consumed 10000 NFE to obtain the better competitive results. Nevertheless, other compared algorithms need much more computing resources except for MADE. It is worth knowing that the MLBSA, JADE, TLABC, and MADE did not reach the BRMSE values. For standard deviation, the EBLSHADE obtained the best

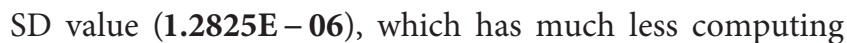
resources than the other selected algorithms, except for MADE.

To further prove the accuracy of the parameters optimized by EBLSHADE, detailed results of the IAEI and IAEP 


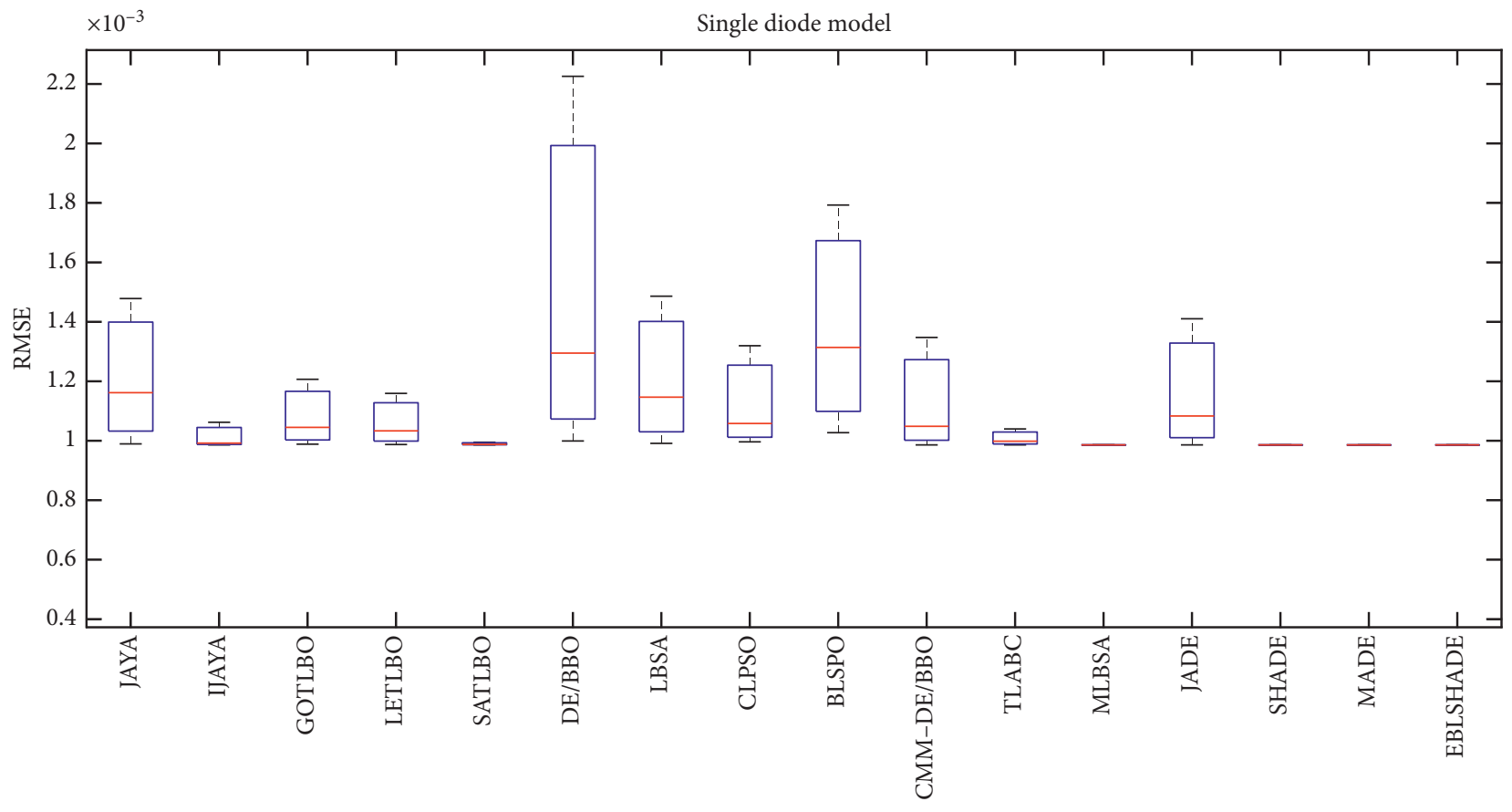

Algorithms

FIgURE 6: The boxplot of the RMSE.

TABle 4: The IAE of EBLSHADE.

\begin{tabular}{|c|c|c|c|}
\hline \multicolumn{2}{|c|}{ Experimental current data } & \multicolumn{2}{|c|}{ Experimental power data } \\
\hline Ic $(V)$ & IAEI $(A)$ & $\operatorname{Pc}(W)$ & IAEP $(W)$ \\
\hline 0.7640876897 & 0.0000876897 & -0.1571728378 & 0.0000180378 \\
\hline 0.7626630749 & 0.0006630749 & 0.0984598030 & 0.0000856030 \\
\hline 0.7613552985 & 0.0008552985 & -0.0447676916 & 0.0000502916 \\
\hline 0.7601539848 & 0.0003460152 & 0.0043328777 & 0.0000019723 \\
\hline 0.7590552049 & 0.0009447951 & 0.0490349662 & 0.0000610338 \\
\hline 0.7580423432 & 0.0009576568 & 0.0898280177 & 0.0001134823 \\
\hline 0.7570916538 & 0.0000916538 & 0.1270399795 & 0.0000153795 \\
\hline 0.7561413663 & 0.0008586337 & 0.1612093393 & 0.0001830607 \\
\hline 0.7550868757 & 0.0004131243 & 0.1921696099 & 0.0001051401 \\
\hline 0.7536638825 & 0.0003361175 & 0.2203713192 & 0.0000982808 \\
\hline 0.7513909717 & 0.0008909717 & 0.2456297087 & 0.0002912587 \\
\hline 0.7473538572 & 0.0008538572 & 0.2679263578 & 0.0003061078 \\
\hline 0.7401172279 & 0.0016172279 & 0.2866474024 & 0.0006263524 \\
\hline 0.7273822304 & 0.0006177696 & 0.3009180287 & 0.0002555713 \\
\hline 0.7069726557 & 0.0004726557 & 0.3091591424 & 0.0002066924 \\
\hline 0.6752801543 & 0.0002198457 & 0.3099535908 & 0.0001009092 \\
\hline 0.6307582734 & 0.0012417266 & 0.3017547580 & 0.0005940420 \\
\hline 0.5719283577 & 0.0010716423 & 0.2836764654 & 0.0005315346 \\
\hline 0.4996070168 & 0.0006070168 & 0.2557488319 & 0.0003107319 \\
\hline 0.4136487897 & 0.0006487897 & 0.2177860878 & 0.0003415878 \\
\hline 0.3175101072 & 0.0010101072 & 0.1713919559 & 0.0005452559 \\
\hline 0.2121549377 & 0.0001549377 & 0.1171307411 & 0.0000855411 \\
\hline 0.1022513120 & 0.0012486880 & 0.0575981641 & 0.0007033859 \\
\hline-0.0087175392 & 0.0012824608 & -0.0050003805 & 0.0007356195 \\
\hline-0.1255074073 & 0.0025074073 & -0.0732084707 & 0.0014625707 \\
\hline--0.2084723186 & 0.0015276814 & -0.1229986680 & 0.0009013320 \\
\hline
\end{tabular}




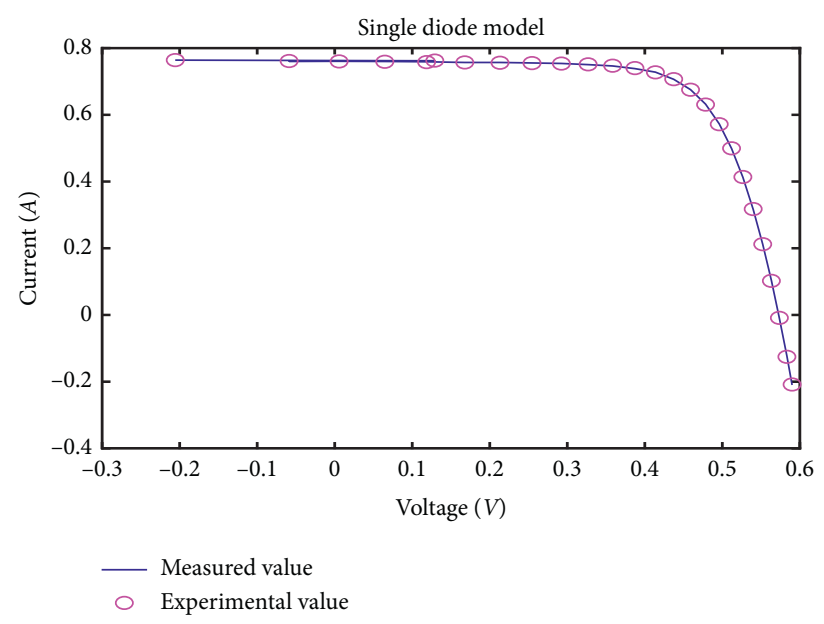

FIgURE 7: The I-V characteristics curve.

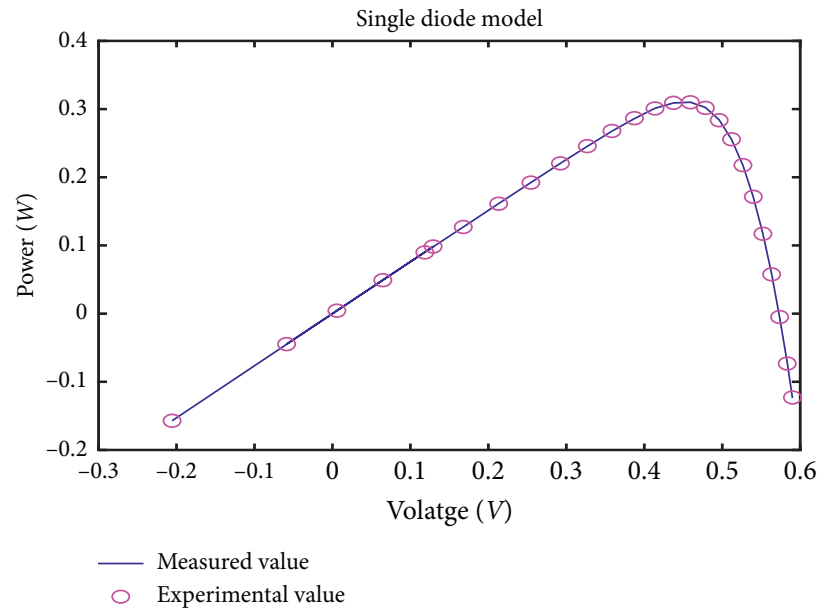

Figure 8: The P-V characteristics curve.

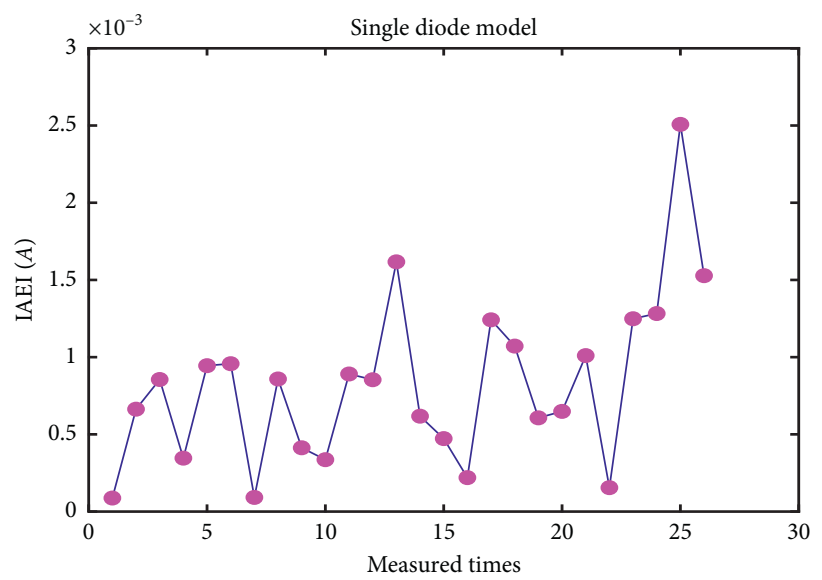

FIgURE 9: The error values of IAEI.

are shown in Table 6. The obtained I-V and P-V curves are shown in Figures 12-15. See references for measurement data.
From Table 6 and Figures 12-15, all IAEI values are lesser than $2.5070 \mathrm{E}-03$ and all IAEP values are lesser than 1.4623E-03, which prove that the EBLSHADE can obtain 


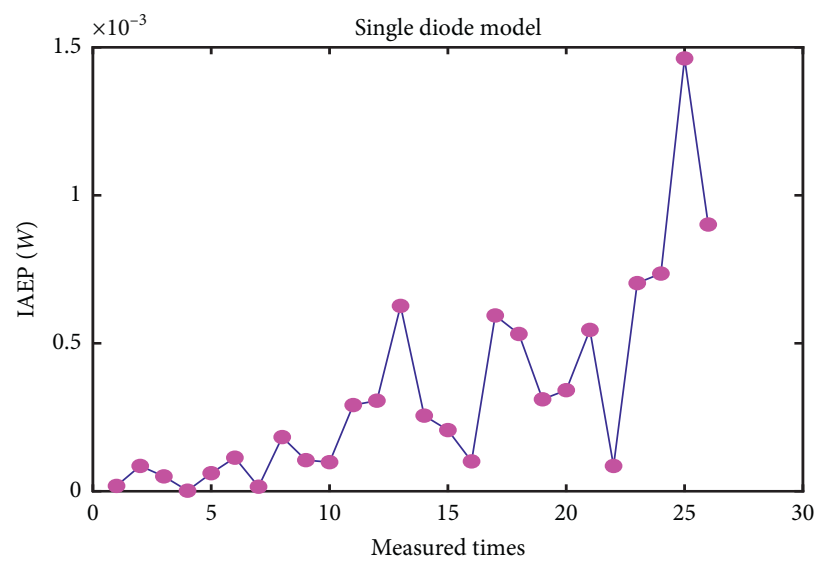

Figure 10: The error values of IAEP.

TABle 5: Comparison results of different algorithms.

\begin{tabular}{|c|c|c|c|c|c|c|c|c|c|c|c|}
\hline Algorithm & $I_{\mathrm{ph}}(A)$ & $I_{\mathrm{sd} 1}(\mu \mathrm{A})$ & $I_{\mathrm{sd} 2}(\mu \mathrm{A})$ & $R_{s}(\Omega)$ & $R_{\mathrm{sh}}(\Omega)$ & $\alpha_{1}$ & $\alpha_{2}$ & BRMSE & MRMSE & SD & NFE \\
\hline JAYA & 0.7601 & 0.0050 & 0.7509 & 0.0367 & 77.8519 & 1.2186 & 1.6447 & $8934 \mathrm{E}-04$ & $.1767 \mathrm{E}-03$ & $1.9356 \mathrm{E}-04$ & 50000 \\
\hline IJAYA & 7601 & .0050 & & .0376 & 77.8519 & & & $9.8293 \mathrm{E}-04$ & $269 E-03$ & & 50000 \\
\hline GOTLBO & 7608 & & 05 & & 56.0753 & & & & & & 20000 \\
\hline LETL & & & & 65 & 54.3021 & & & & & & 50000 \\
\hline & & & & & & & & & & & 50000 \\
\hline $\mathrm{DE} / \mathrm{BB}$ & 7608 & .0012 & & & & & & & & & 50000 \\
\hline BSA & 7606 & 0.298 & 0.2710 & & 60.1 & & & & & & 50000 \\
\hline CLP & & & 0.3 & & & & & & 1.1 & 1.43 & 50000 \\
\hline BLSI & 7608 & & & & & & & & & & 50000 \\
\hline CMM-DE/BBO & & & & & & & & & & & 50000 \\
\hline MLBSA & .7608 & 0.4239 & 0.2401 & 0.0367 & 54.6680 & 1.9075 & 1.4567 & $9.8415 \mathrm{E}-04$ & 1.05 & $1.5500 \mathrm{E}-04$ & 50000 \\
\hline TLABC & & & & & & & & & & & 50000 \\
\hline I & 7608 & & & & & & 1.4669 & 9.83 & & $3.8100 \mathrm{E}-04$ & 20000 \\
\hline SHADE & .7608 & 0.2260 & 0.7494 & 0.0367 & 55.4854 & 1.4510 & 2.0000 & $9.8248 E-04$ & $1.0867 \mathrm{E}-03$ & $2.6000 \mathrm{E}-05$ & 20000 \\
\hline MADE & .7608 & 0.7394 & 0.2246 & 0.0368 & 55.4329 & 1.9963 & 1.4505 & $9.8261 \mathrm{E}-04$ & $9.8608 \mathrm{E}-04$ & $8.0200 \mathrm{E}-05$ & 10000 \\
\hline EBLSHADE & 0.7608 & 0.3886 & 0.6889 & 0.0364 & 53.6878 & 1.4866 & 1.5251 & $9.8295 E-04$ & $9.8574 \mathrm{E}-04$ & $1.2825 E-06$ & 10000 \\
\hline
\end{tabular}

the optimized parameters with higher accuracy. The experimental results show that the experimental data by EBLSHADE are highly in agreement with the measured data. So, the EBLSHADE is regarded as a significant algorithm to optimize the parameters of DDM.

5.4.3. Experimental Results of PVM. For the PV models, to further prove the performance of the EBLSHADE, photowatt-PWP201, STM6-40/36, and STP6-120/36 are used in here. The experimental results of the PVMs are shown in Tables 7-9. The boxplot of RMSE are presented in Figures 16-18.

From Table 7 and Figure 16 on the photowatt-PWP201 model, all compared algorithms can obtain the same BRMSE (2.4251E-03), while the EBLSHADE only consumed the least 5,000 NFE. Especially for the SHADE, MADE, PGJAYA, and EBLSHADE, the best, worst, and mean RMSE values are the same value $(2.4251 \mathrm{E}-03)$, which indicate that the SHADE, MADE, PGJAYA, and EBLSHADE take on better stability. For standard deviation, the EBLSHADE obtained the second best standard deviation value
$(2.8821 \mathrm{E}-17)$, which is close to the best standard deviation value $(2.0700 E-17)$. Although the EBLSHADE did no't obtain the best standard deviation value, it only consumed 5,000 NFE to obtain the better optimization results.

From Table 7 and Figure 17 on the STM6-40/36 model, except for IJAYA and JADE, the other compared algorithms can get the same BRMSE value (1.7298E - 03). Especially for MADE and EBLSHADE, the best, worst, and mean RMSE values are the same value $(\mathbf{1 . 7 2 9 8 E}-\mathbf{0 3})$, which indicate that the MADE and EBLSHADE take on better stability. For standard deviation, the EBLSHADE obtained the best standard deviation value $(6.40591 E-14)$. The max NFE of EBLSHADE is $10,000 \mathrm{NFE}$, which utilizes less computational resources compared to other compared algorithms except for MADE. Although the EBLSHADE is not less NFE for the STM6-40/36 model, it consumed 10,000 NFE to obtain the better optimization results.

From Table 8 and Figure 18 on the STP6-120/36 model, except for IJAYA and JADE, the other compared algorithms can get the same BRMSE value $(1.6601 \mathrm{E}-02)$. Especially for SATLBO, MADE, and EBLSHADE, the best, worst, and mean RMSE values are the same value $(1.6601 \mathrm{E}-02)$, which 
TABle 6: The IAE of EBLSHADE.

\begin{tabular}{|c|c|c|c|}
\hline \multicolumn{2}{|c|}{ Experimental current data } & \multicolumn{2}{|c|}{ Experimental power data } \\
\hline Ic $(V)$ & IAEI $(A)$ & Pc $(W)$ & $\operatorname{IAEP}(W)$ \\
\hline 0.7640899318 & 0.0000899318 & -0.1571732990 & 0.0000184990 \\
\hline 0.7626644760 & 0.0006644760 & 0.0984599838 & 0.0000857838 \\
\hline 0.7613559283 & 0.0008559283 & -0.0447677286 & 0.0000503286 \\
\hline 0.7601539095 & 0.0003460905 & 0.0043328773 & 0.0000019727 \\
\hline 0.7590544948 & 0.0009455052 & 0.0490349204 & 0.0000610796 \\
\hline 0.7580410765 & 0.0009589235 & 0.0898278676 & 0.0001136324 \\
\hline 0.7570899338 & 0.0000899338 & 0.1270396909 & 0.0000150909 \\
\hline 0.7561393418 & 0.0008606582 & 0.1612089077 & 0.0001834923 \\
\hline 0.7550847711 & 0.0004152289 & 0.1921690742 & 0.0001056758 \\
\hline 0.7536620011 & 0.0003379989 & 0.2203707691 & 0.0000988309 \\
\hline 0.7513896669 & 0.0008896669 & 0.2456292821 & 0.0002908321 \\
\hline 0.7473534622 & 0.0008534622 & 0.2679262162 & 0.0003059662 \\
\hline 0.7401179218 & 0.0016179218 & 0.2866476711 & 0.0006266211 \\
\hline 0.7273839415 & 0.0006160585 & 0.3009187366 & 0.0002548634 \\
\hline 0.7069749599 & 0.0004749599 & 0.3091601500 & 0.0002077000 \\
\hline 0.6752824593 & 0.0002175407 & 0.3099546488 & 0.0000998512 \\
\hline 0.6307600021 & 0.0012399979 & 0.3017555850 & 0.0005932150 \\
\hline 0.5719291625 & 0.0010708375 & 0.2836768646 & 0.0005311354 \\
\hline 0.4996068718 & 0.0006068718 & 0.2557487577 & 0.0003106577 \\
\hline 0.4136480065 & 0.0006480065 & 0.2177856754 & 0.0003411754 \\
\hline 0.3175090962 & 0.0010090962 & 0.1713914101 & 0.0005447101 \\
\hline 0.2121541459 & 0.0001541459 & 0.1171303040 & 0.0000851040 \\
\hline 0.1022510195 & 0.0012489805 & 0.0575979993 & 0.0007035507 \\
\hline-0.0087176082 & 0.0012823918 & -0.0050004200 & 0.0007355800 \\
\hline-0.1255069400 & 0.0025069400 & -0.0732081981 & 0.0014622981 \\
\hline-0.2084723257 & 0.0015276743 & -0.1229986722 & 0.0009013278 \\
\hline
\end{tabular}

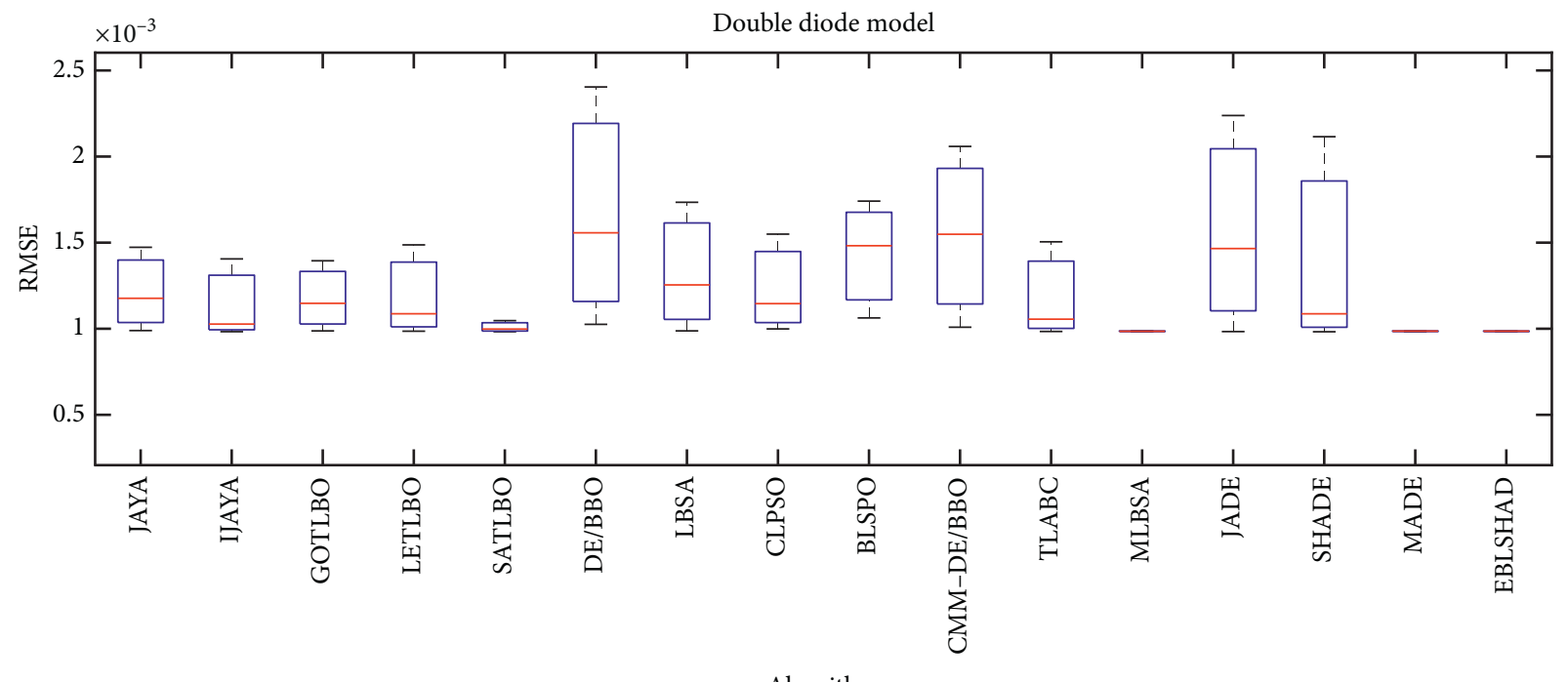

FIgURE 11: The boxplot of RMSE.

indicate that the SATLBO, MADE, and EBLSHADE take on better stability. For standard deviation, the EBLSHADE obtained the best standard deviation value $(8.0544 \mathrm{E}-16)$. The max NFE of EBLSHADE is 15,000 NFE, which are much less computational resources than the other compared algorithms except for MADE. Although the EBLSHADE is not less NFE for the STP6-120/36 model, it consumed 15,000 NFE to obtain the better optimization results.

Therefore, it is clear that the EBLSHADE can effectively and consistently provide better results of integrating the RMSE and NFE considerations by comparing with other methods. 


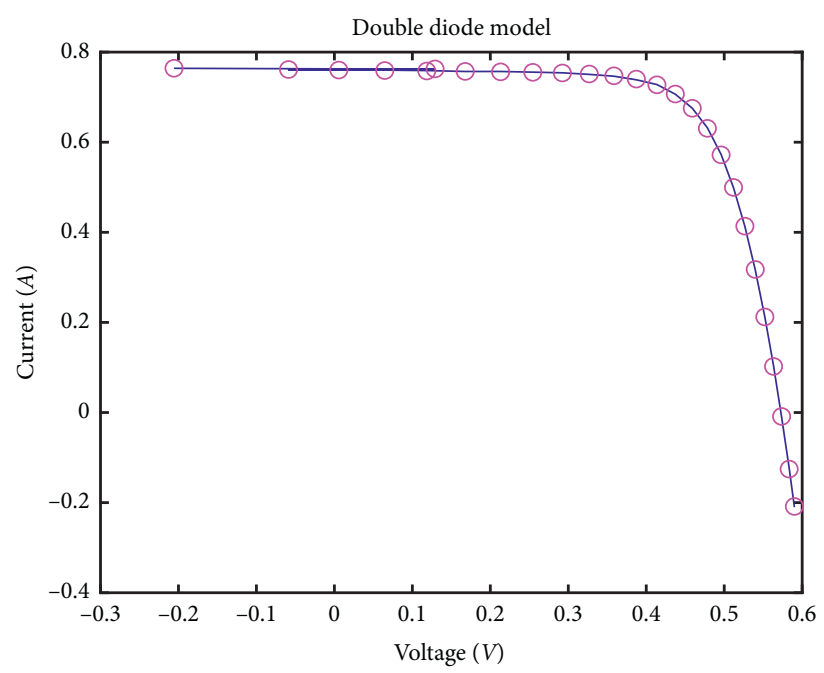

- Measured value

- Experimental value

Figure 12: The I-V curve.

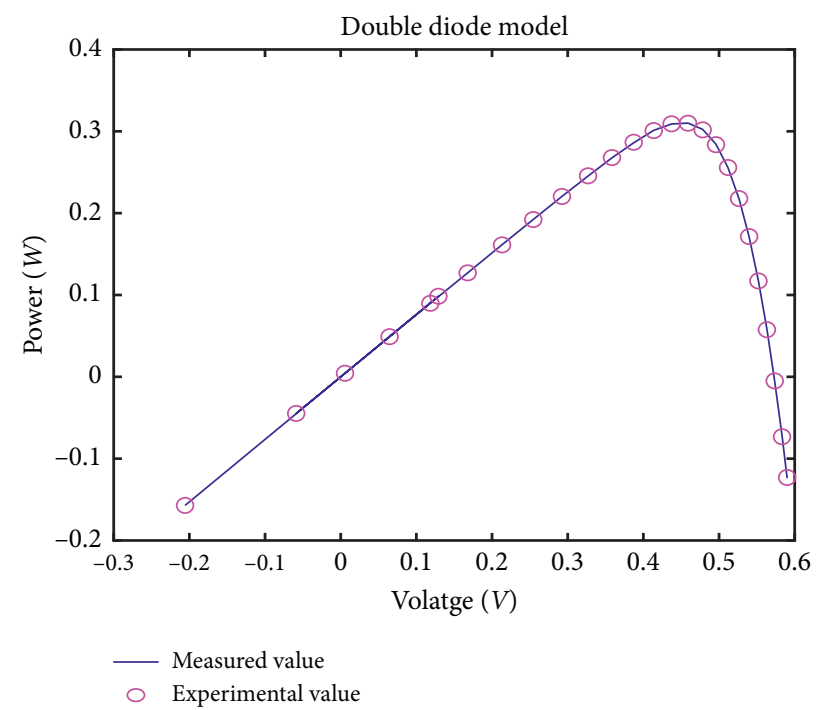

Figure 13: The P-V curve.

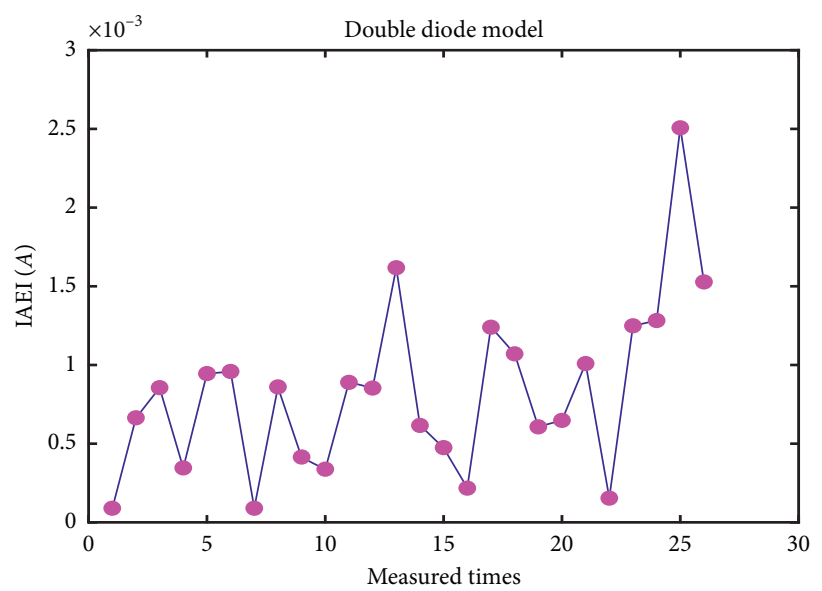

Figure 14: The error of IAEI. 


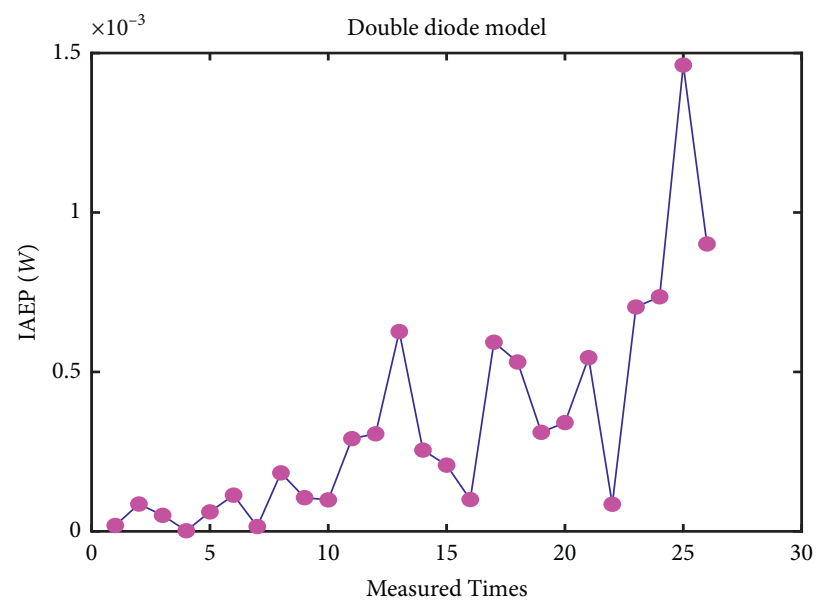

Figure 15: The error of IAEP.

TABLE 7: Comparison results on the photowatt-PWP202 model.

\begin{tabular}{lccccccccc}
\hline Algorithm & $I_{\mathrm{ph}}(A)$ & $I_{d}(\mu \mathrm{A})$ & $R_{s}(\Omega)$ & $R_{\mathrm{sh}}(\Omega)$ & $\alpha$ & BRMSE & MRMSE & SD & NFE \\
\hline IJAYA & 1.0305 & 3.4703 & 1.2016 & 977.3752 & 48.6298 & $\mathbf{2 . 4 2 5 1 E}-\mathbf{0 3}$ & $2.4289 \mathrm{E}-03$ & $3.7755 \mathrm{E}-06$ & 50000 \\
GOTLBO & 1.0305 & 3.4991 & 1.2008 & 989.6889 & 48.6611 & $\mathbf{2 . 4 2 5 1 E}-\mathbf{0 3}$ & $2.4419 \mathrm{E}-03$ & $1.3800 \mathrm{E}-05$ & 10000 \\
SATLBO & 1.0305 & 3.4827 & 1.2013 & 982.4038 & 48.6433 & $\mathbf{2 . 4 2 5 1 E}-\mathbf{0 3}$ & $2.4254 \mathrm{E}-03$ & $7.4100 \mathrm{E}-07$ & 50000 \\
TLABC & 1.0306 & 3.4715 & 1.2017 & 972.9357 & 48.6313 & $\mathbf{2 . 4 2 5 1 E}-\mathbf{0 3}$ & $2.4265 \mathrm{E}-03$ & $4.0000 \mathrm{E}-06$ & 50000 \\
MLBSA & 1.0305 & 3.4823 & 1.2013 & 981.9823 & 48.6238 & $\mathbf{2 . 4 2 5 1 E}-\mathbf{0 3}$ & $2.4251 \mathrm{E}-03$ & $4.3400 \mathrm{E}-08$ & 50000 \\
JADE & 1.0305 & 3.4823 & 1.2012 & 982.3236 & 48.6238 & $\mathbf{2 . 4 2 5 1 E}-\mathbf{0 3}$ & $2.4343 \mathrm{E}-03$ & $1.1900 \mathrm{E}-05$ & 10000 \\
SHADE & 1.0305 & 3.4823 & 1.2013 & 981.9822 & 48.6428 & $\mathbf{2 . 4 2 5 1 E}-\mathbf{0 3}$ & $\mathbf{2 . 4 2 5 1 E}-\mathbf{0 3}$ & $1.9300 \mathrm{E}-16$ & 10000 \\
MADE & 1.0305 & 3.4823 & 1.2013 & 981.9823 & 48.6428 & $\mathbf{2 . 4 2 5 1 E}-\mathbf{0 3}$ & $\mathbf{2 . 4 2 5 1 E}-\mathbf{0 3}$ & $\mathbf{2 . 0 7 0 0 E}-\mathbf{1 7}$ & 10000 \\
PGJAYA & 1.0305 & 3.4818 & 1.2013 & 981.8545 & 48.6424 & $\mathbf{2 . 4 2 5 1 E}-\mathbf{0 3}$ & $\mathbf{2 . 4 2 5 1 E}-\mathbf{0 3}$ & $3.0700 \mathrm{E}-07$ & 50000 \\
EBLSHADE & 1.0305 & 3.4823 & 1.2013 & 981.9825 & 48.6428 & $\mathbf{2 . 4 2 5 1 E}-\mathbf{0 3}$ & $\mathbf{2 . 4 2 5 1 E}-\mathbf{0 3}$ & $2.8821 \mathrm{E}-17$ & $\mathbf{5 0 0 0}$ \\
\hline
\end{tabular}

TABLE 8: Comparison results on the STM6-40/36 model.

\begin{tabular}{lccccccccc}
\hline Algorithm & $I_{\mathrm{ph}}(A)$ & $I_{d}(\mu \mathrm{A})$ & $R_{s}(\Omega)$ & $R_{\mathrm{sh}}(\Omega)$ & $\alpha$ & BRMSE & MRMSE & SD \\
\hline IJAYA & 1.6637 & 1.8353 & 0.0040 & 15.9449 & 1.5263 & $1.7548 \mathrm{E}-03$ & $1.9305 \mathrm{E}-04$ & $1.9100 \mathrm{E}-04$ & 50000 \\
GOTLBO & 1.6639 & 1.7387 & 0.0043 & 15.9283 & 1.5203 & $\mathbf{1 . 7 2 9 8 E}-\mathbf{0 3}$ & $4.2347 \mathrm{E}-03$ & $2.6800 \mathrm{E}-03$ & 50000 \\
SATLBO & 1.6639 & 1.7387 & 0.0043 & 15.9283 & 1.5203 & $\mathbf{1 . 7 2 9 8 E}-\mathbf{0 3}$ & $1.7298 \mathrm{E}-03$ & $1.2200 \mathrm{E}-08$ & 50000 \\
TLABC & 1.6639 & 1.7389 & 0.0043 & 15.9290 & 1.5203 & $\mathbf{1 . 7 2 9 8 E}-\mathbf{0 3}$ & $2.1827 \mathrm{E}-03$ & $9.2200 \mathrm{E}-04$ & 50000 \\
MLBSA & 1.6639 & 1.7387 & 0.0043 & 15.9283 & 1.5203 & $\mathbf{1 . 7 2 9 8 E}-\mathbf{0 3}$ & $1.7382 \mathrm{E}-03$ & $1.4500 \mathrm{E}-05$ & 50000 \\
JADE & 1.6638 & 1.7946 & 0.0042 & 16.0190 & 1.5238 & $1.7324 \mathrm{E}-03$ & $2.1308 \mathrm{E}-03$ & $4.0500 \mathrm{E}-04$ & 15000 \\
SHADE & 1.6639 & 1.7386 & 0.0043 & 15.9282 & 1.5203 & $\mathbf{1 . 7 2 9 8 E}-\mathbf{0 3}$ & $1.7306 \mathrm{E}-03$ & $2.4400 \mathrm{E}-06$ & 10000 \\
MADE & 1.6639 & 1.7387 & 0.0043 & 15.9283 & 1.5203 & $\mathbf{1 . 7 2 9 8 E}-\mathbf{0 3}$ & $\mathbf{1 . 7 2 9 8 E}-\mathbf{0 3}$ & $8.4900 \mathrm{E}-14$ & $\mathbf{7 0 0 0}$ \\
PGJAYA & 1.6639 & 1.7389 & 0.0043 & 15.9290 & 1.5203 & $\mathbf{1 . 7 2 9 8 E}-\mathbf{0 3}$ & $1.7299 \mathrm{E}-03$ & $7.8200 \mathrm{E}-08$ & 50000 \\
EBLSHADE & 1.6639 & 1.7387 & 0.0043 & 15.9283 & 1.5203 & $\mathbf{1 . 7 2 9 8 E}-\mathbf{0 3}$ & $\mathbf{1 . 7 2 9 8 E}-\mathbf{0 3}$ & $\mathbf{6 . 4 0 5 9 1 E}-\mathbf{1 4}$ & 10000 \\
\hline
\end{tabular}

TABLE 9: Comparison results on the STP6-120/36 model.

\begin{tabular}{lccccccccc}
\hline Algorithm & $I_{\mathrm{ph}}(A)$ & $I_{d}(\mu \mathrm{A})$ & $R_{s}(\Omega)$ & $R_{\mathrm{sh}}(\Omega)$ & $\alpha$ & BRMSE & MRMSE & SD & NFE \\
\hline IJAYA & 7.4672 & 2.2536 & 0.0046 & 27.5925 & 1.2571 & $1.6731 \mathrm{E}-02$ & $1.6891 \mathrm{E}-02$ & $1.1200 \mathrm{E}-04$ & 50000 \\
GOTLBO & 7.4725 & 2.3350 & 0.0046 & 22.2199 & 1.2601 & $\mathbf{1 . 6 6 0 1 E}-\mathbf{0 2}$ & $2.9588 \mathrm{E}-02$ & $3.0500 \mathrm{E}-02$ & 50000 \\
SATLBO & 7.4725 & 2.3350 & 0.0046 & 22.2199 & 1.2601 & $\mathbf{1 . 6 6 0 1 E}-\mathbf{0 2}$ & $\mathbf{1 . 6 6 0 1 E}-\mathbf{0 2}$ & $2.0200 \mathrm{E}-09$ & 50000 \\
TLABC & 7.4725 & 2.3349 & 0.0046 & 22.2117 & 1.2601 & $\mathbf{1 . 6 6 0 1 E}-\mathbf{0 2}$ & $1.6963 \mathrm{E}-02$ & $9.4700 \mathrm{E}-04$ & 50000 \\
MLBSA & 7.4725 & 2.3350 & 0.0046 & 22.2199 & 1.2601 & $\mathbf{1 . 6 6 0 1 E}-\mathbf{0 2}$ & $1.6731 \mathrm{E}-02$ & $3.0100 \mathrm{E}-04$ & 50000 \\
JADE & 7.4645 & 3.4139 & 0.0044 & 1439.6969 & 1.2926 & $1.7430 \mathrm{E}-02$ & $2.7994 \mathrm{E}-02$ & $5.6200 \mathrm{E}-03$ & 15000 \\
SHADE & 7.4725 & 2.3353 & 0.0046 & 22.2169 & 1.2601 & $\mathbf{1 . 6 6 0 1 E}-\mathbf{0 2}$ & $2.4820 \mathrm{E}-02$ & $1.3900 \mathrm{E}-02$ & 10000 \\
MADE & 7.4725 & 2.3353 & 0.0046 & 22.2199 & 1.2601 & $\mathbf{1 . 6 6 0 1 E}-\mathbf{0 2}$ & $\mathbf{1 . 6 6 0 1 E}-\mathbf{0 2}$ & $1.6900 \mathrm{E}-15$ & 7000 \\
PGJAYA & 7.4725 & 0.0000 & 0.0046 & 22.2184 & 1.2601 & $\mathbf{1 . 6 6 0 1 E}-\mathbf{0 2}$ & $1.6602 \mathrm{E}-02$ & $2.5700 \mathrm{E}-06$ & 50000 \\
EBLSHADE & 7.4725 & 2.3350 & 0.0046 & 22.2199 & 1.2601 & $\mathbf{1 . 6 6 0 1 E}-\mathbf{0 2}$ & $\mathbf{1 . 6 6 0 1 E}-\mathbf{0 2}$ & $\mathbf{8 . 0 5 4 4 E}-\mathbf{1 6}$ & 15000 \\
\hline
\end{tabular}


TABLE 10: The IAE of the EBLSHADE on the photowatt-PWP201 model.

\begin{tabular}{|c|c|c|c|}
\hline \multicolumn{2}{|c|}{ Experimental current data } & \multicolumn{2}{|c|}{ Experimental power data } \\
\hline Ic $(V)$ & IAEI $(A)$ & $\operatorname{Pc}(W)$ & IAEP $(W)$ \\
\hline 1.0291191606 & 0.0023808394 & 0.1284340712 & 0.0002971288 \\
\hline 1.0273810726 & 0.0026189274 & 1.8588405747 & 0.0047384253 \\
\hline 1.0257417965 & 0.0002582035 & 3.4373633343 & 0.0008652657 \\
\hline 1.0241071545 & 0.0021071545 & 4.8770030911 & 0.0100346911 \\
\hline 1.0222918044 & 0.0042918044 & 6.1887501253 & 0.0259817253 \\
\hline 1.0199306808 & 0.0044306808 & 7.3806263783 & 0.0320621783 \\
\hline 1.0163631056 & 0.0023631056 & 8.4550230394 & 0.0196584394 \\
\hline 1.0104961513 & 0.0004961513 & 9.4074160197 & 0.0046190197 \\
\hline 1.0006289697 & 0.0028710303 & 10.2227257430 & 0.0293313070 \\
\hline 0.9845483784 & 0.0034516216 & 10.8742383845 & 0.0381228155 \\
\hline 0.9595216759 & 0.0034783241 & 11.3240829147 & 0.0410504853 \\
\hline 0.9228388177 & 0.0026611823 & 11.5289330659 & 0.0332458841 \\
\hline 0.8725996624 & 0.0000996624 & 11.4512126291 & 0.0013078791 \\
\hline 0.8072742631 & 0.0002257369 & 11.0582850383 & 0.0030922117 \\
\hline 0.7283364774 & 0.0018364774 & 10.3584742145 & 0.0261185645 \\
\hline 0.6371379993 & 0.0026379993 & 9.3656100213 & 0.0387772713 \\
\hline 0.5362130625 & 0.0017130625 & 8.1153702155 & 0.0259265155 \\
\hline 0.4295113243 & 0.0020113243 & 6.6707833287 & 0.0312380787 \\
\hline 0.3187744822 & 0.0002744822 & 5.0662509687 & 0.0043623187 \\
\hline 0.2073895062 & 0.0011104938 & 3.3644592206 & 0.0180154294 \\
\hline 0.0961671715 & 0.0048328285 & 1.5890759579 & 0.0798581421 \\
\hline-0.0083253863 & 0.0003253863 & -0.1398556671 & 0.0054660671 \\
\hline-0.1109364828 & 0.0000635172 & -1.8914559374 & 0.0010829626 \\
\hline-0.2092472658 & 0.0002472658 & -3.6156462796 & 0.0042725796 \\
\hline-0.3008635866 & 0.0021364134 & -5.2616528348 & 0.0373626652 \\
\hline
\end{tabular}

TABLE 11: The IAE of the EBLSHADE on the STM6-40/36 model.

\begin{tabular}{llrr}
\hline \multicolumn{2}{c}{ Experimental current data } & \multicolumn{2}{c}{ Experimental power data } \\
Ic $(V)$ & IAEI $(A)$ & Pc $(W)$ & 0.0000000000 \\
\hline 1.6634582480 & 0.0004582480 & 0.1962637713 & 0.0000000000 \\
1.6632522991 & 0.0002522991 & 3.7124151139 & 0.0000297713 \\
1.6595507885 & 0.0014492115 & 8.9873722902 & 0.0032418861 \\
1.6539146651 & 0.0009146651 & 11.9831082348 & 0.0049702902 \\
1.6505658726 & 0.0005658726 & 15.9277677859 & 0.0041082348 \\
1.6454305564 & 0.0004305564 & 18.9987161328 & 0.0041677859 \\
1.6392334886 & 0.0007665114 & 20.5847794140 & 0.0088838672 \\
1.6337126519 & 0.0022873481 & 21.7568107486 & 0.0288205860 \\
1.6272857703 & 0.0017142297 & 22.8020378709 & 0.0229192514 \\
1.6183135466 & 0.0006864534 & 23.8539796241 & 0.0096721291 \\
1.6030900285 & 0.0060900285 & 24.6569627345 & 0.0906196241 \\
1.5815883730 & 0.0005883730 & 25.2942218610 & 0.0091727345 \\
1.5423306013 & 0.0003306013 & 25.4191291363 & 0.0054218610 \\
1.5211926473 & 0.0028073527 & 25.4563270155 & 0.0469108637 \\
1.4991947595 & 0.0008052405 & 25.4427656382 & 0.0136729845 \\
1.4852752854 & 0.0002752854 & 25.3851317253 & 0.0047156382 \\
1.4656542567 & 0.0006542567 & 24.8517256151 & 0.0113317253 \\
1.3875893699 & 0.0004106301 & 21.3389058008 & 0.0073543849 \\
1.1183912894 & 0.0003912894 & -0.0005220220 & 0.0074658008 \\
-0.0000248345 & 0.0000248345 & & 0.0005220220 \\
\hline
\end{tabular}

To show the accuracy of the parameters optimized by EBLSHADE, detailed results of the IAEI and IAEP for three PVM are shown in Tables 10-12. The obtained I-V and P-V curves are given in Figures 19-30. See references for measurement data.
From Tables 10-12 and Figures 19-30, all IAEI values are lesser than $\mathbf{4 . 8 3 2 9 E}-\mathbf{0 3}$ and all IAEP values are lesser than 3.7363E-02 for the photowatt-PWP201 PV model. For the STM6-40/36 PV model, all IAEI values are lesser than 6.0901E-03 and all IAEP values are lesser than 9.0620E- 
TABle 12: The IAE of the EBLSHADE on the STP6-120/36 model.

\begin{tabular}{|c|c|c|c|}
\hline \multicolumn{2}{|c|}{ Experimental current data } & \multicolumn{2}{|c|}{ Experimental power data } \\
\hline Ic $(V)$ & IAEI $(A)$ & $\operatorname{Pc}(W)$ & IAEP $(W)$ \\
\hline 0.0022826059 & 0.0022826059 & 0.0438488585 & 0.0438488585 \\
\hline 3.8333471928 & 0.0033471928 & 67.6585779533 & 0.0590779533 \\
\hline 4.2673375798 & 0.0226624202 & 74.2943472636 & 0.3945527364 \\
\hline 4.5411485436 & 0.0188514564 & 78.3348123769 & 0.3251876231 \\
\hline 4.7844002096 & 0.0055997904 & 81.8132435841 & 0.0957564159 \\
\hline 5.0855780663 & 0.0155780663 & 85.9462693208 & 0.2632693208 \\
\hline 5.2748224588 & 0.0048224588 & 88.4060244100 & 0.0808244100 \\
\hline 5.7825974772 & 0.0325974772 & 94.4876427780 & 0.5326427780 \\
\hline 6.0443179793 & 0.0443179793 & 97.1926331064 & 0.7126331064 \\
\hline 6.3471209075 & 0.0128790925 & 99.7132694563 & 0.2023305437 \\
\hline 6.5665499123 & 0.0134500877 & 101.0592031502 & 0.2069968498 \\
\hline 6.8136110536 & 0.0163889464 & 101.7272130305 & 0.2446869695 \\
\hline 6.9577097235 & 0.0122902765 & 101.4434077693 & 0.1791922307 \\
\hline 7.0875758039 & 0.0124241961 & 100.4309491411 & 0.1760508589 \\
\hline 7.2173848761 & 0.0126151239 & 98.0842604665 & 0.1714395335 \\
\hline 7.2839995878 & 0.0060004122 & 95.8574345754 & 0.0789654246 \\
\hline 7.3313455009 & 0.0086544991 & 93.4013416819 & 0.1102583181 \\
\hline 7.3631832243 & 0.0068167757 & 91.0089446520 & 0.0842553480 \\
\hline 7.3959998685 & 0.0159998685 & 87.3467584474 & 0.1889584474 \\
\hline 7.4203157738 & 0.0103157738 & 82.8849271934 & 0.1152271934 \\
\hline 7.4390898341 & 0.0009101659 & 76.7714070882 & 0.0093929118 \\
\hline 7.4467621170 & 0.0267621170 & 72.5314630191 & 0.2606630191 \\
\hline 7.4525404203 & 0.0025404203 & 67.5200162083 & 0.0230162083 \\
\hline 7.4709794084 & 0.0090205916 & 0.0000000000 & 0.0000000000 \\
\hline
\end{tabular}

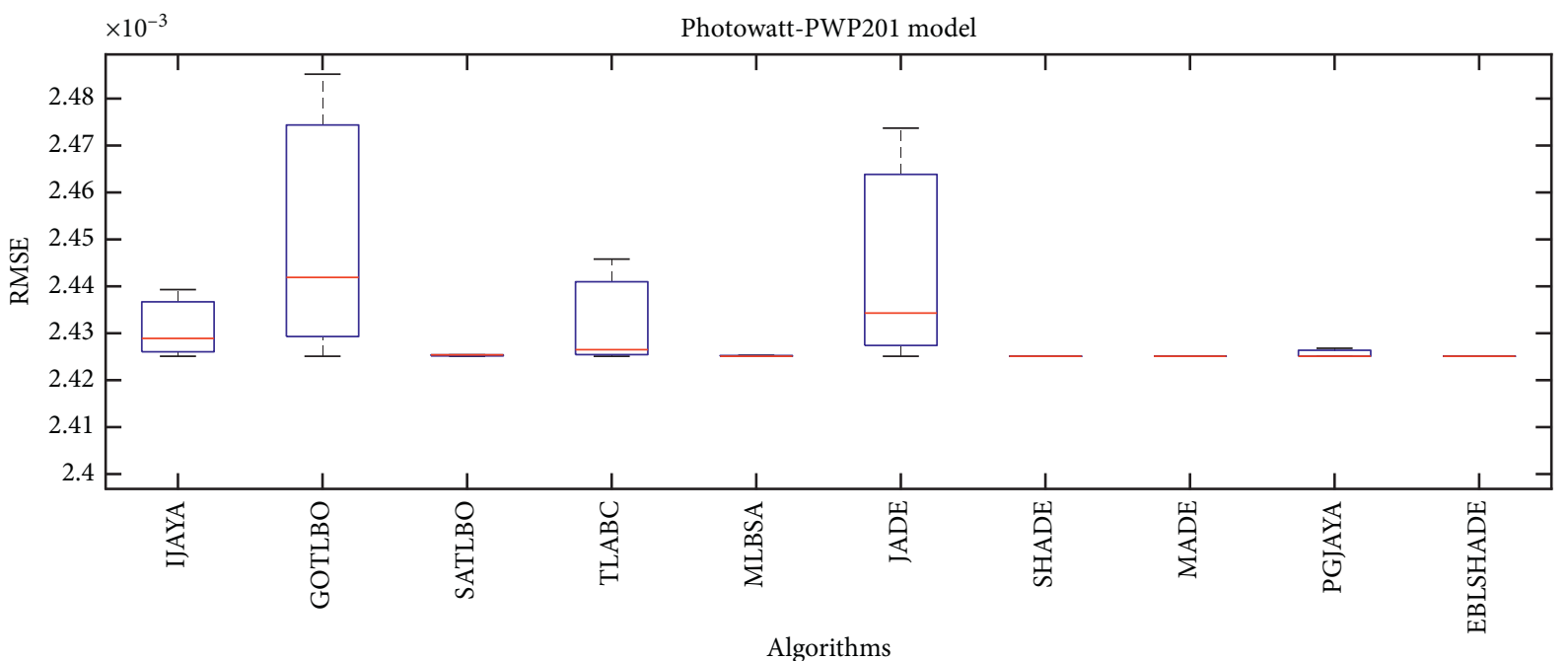

Figure 16: The boxplot of RMSE on the photowatt-PWP201 model.

01. For the STP6-120/36 PV model, all IAEI values are lesser than 4.4318E-02 and all IAEP values are lesser than 7.1264E-01. In general, the EBLSHADE can effectively optimize parameters of the PV models with higher accuracy. It is also clear that the experimental data by EBLSHADE are highly in agreement with the measured data. Therefore, the EBLSHADE is considered as a significant method to optimize the parameters of the PV models.
5.4.4. Discussion of the Results. As the experiment results are given in Section 5.3, the EBLSHADE is applied in the parameter optimization of PV models, respectively. The superiority of the EBLSHADE has been proved by comparing with some other algorithms. It can be seen from the I-V curves and P-V curves that the experimental results of EBLSHADE are highly in agreement with the measured data for all datasets, which effectively reflects the optimized 


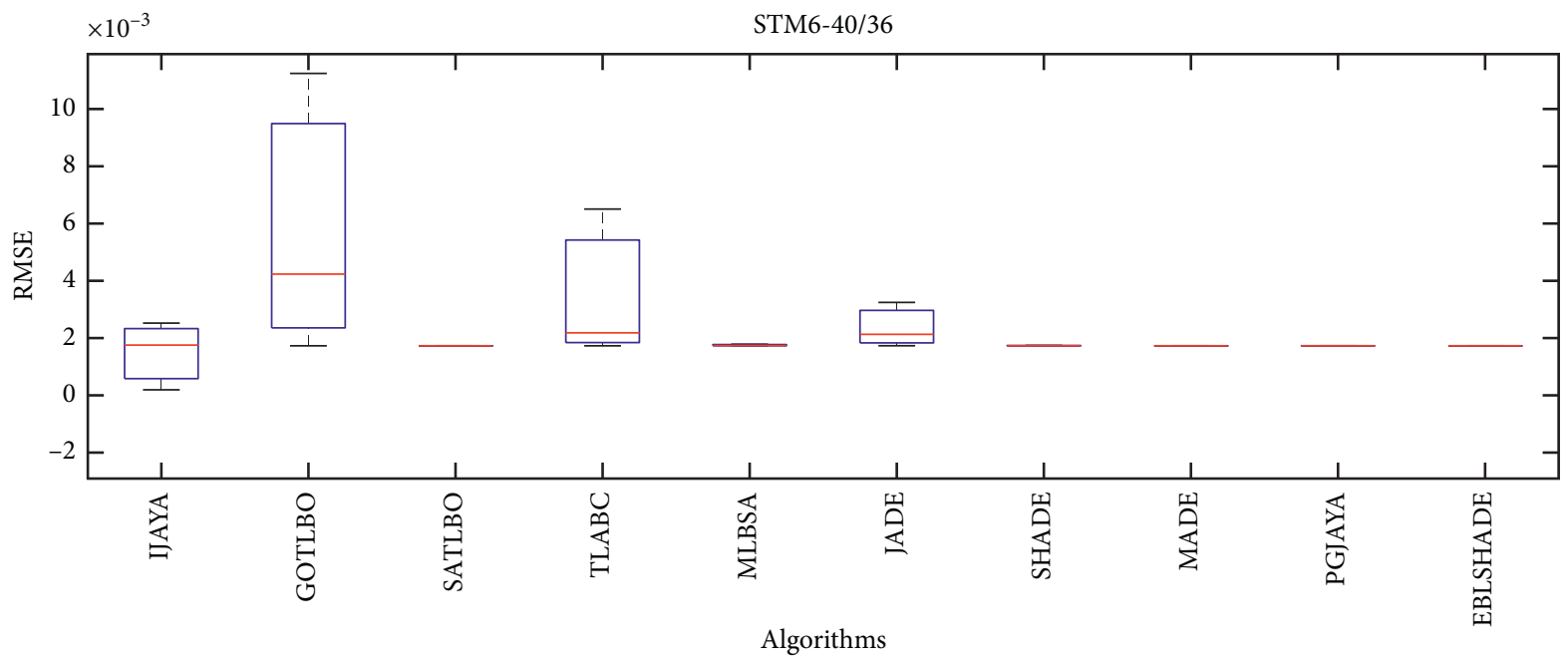

FIgURE 17: The boxplot of RMSE on the STM6-40/36 model.

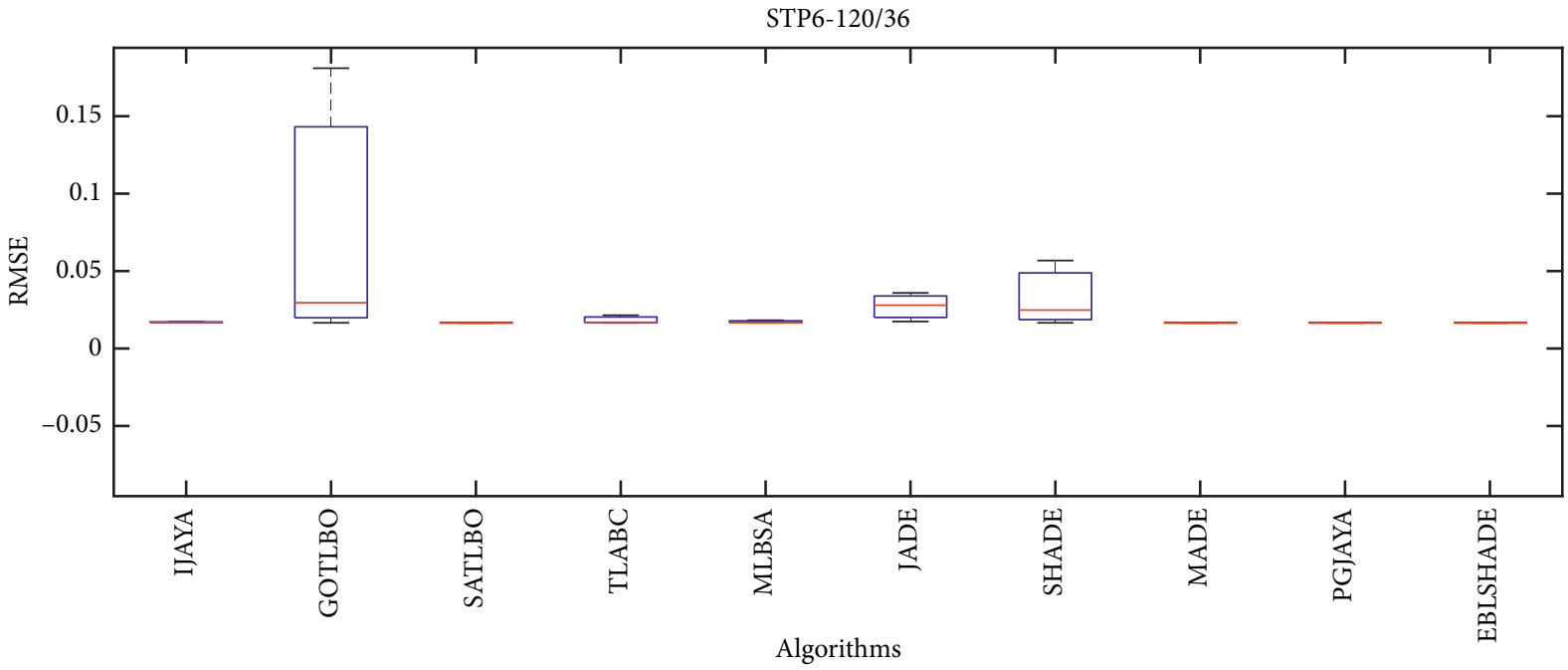

Figure 18: The boxplot of RMSE on the STP6-120/36 model.

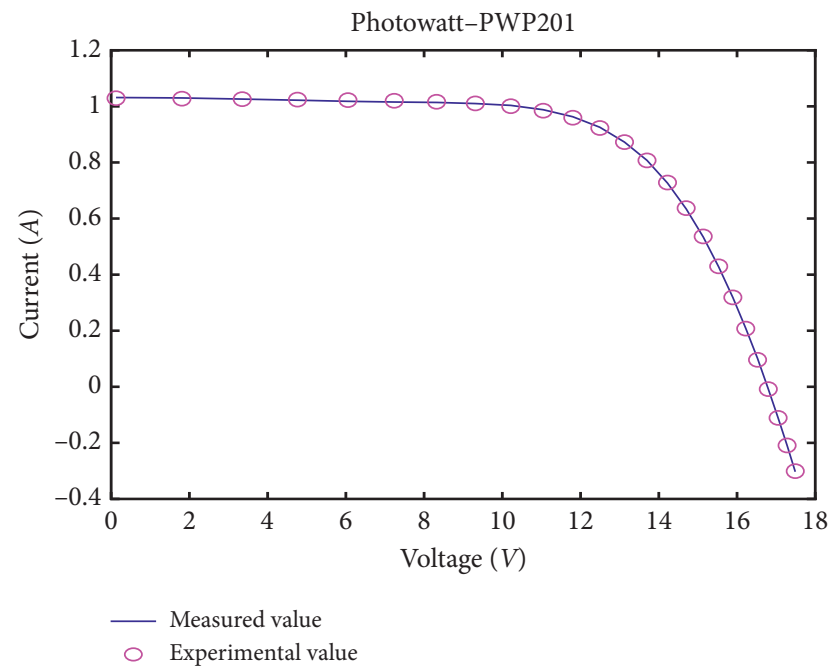

FIgUre 19: The I-V on the photowatt-PWP201 model.

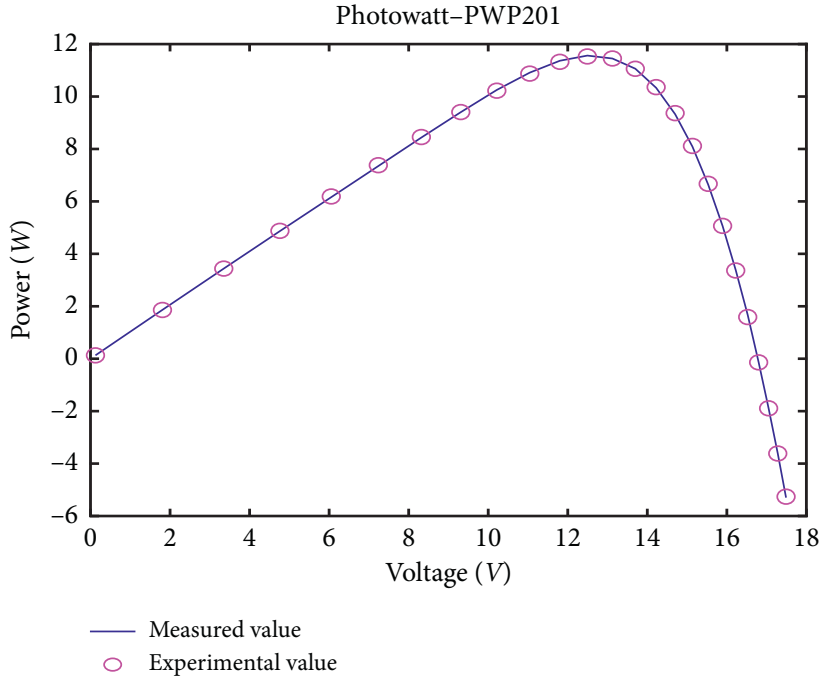

Figure 20: The P-V on the photowatt-PWP201 model. 


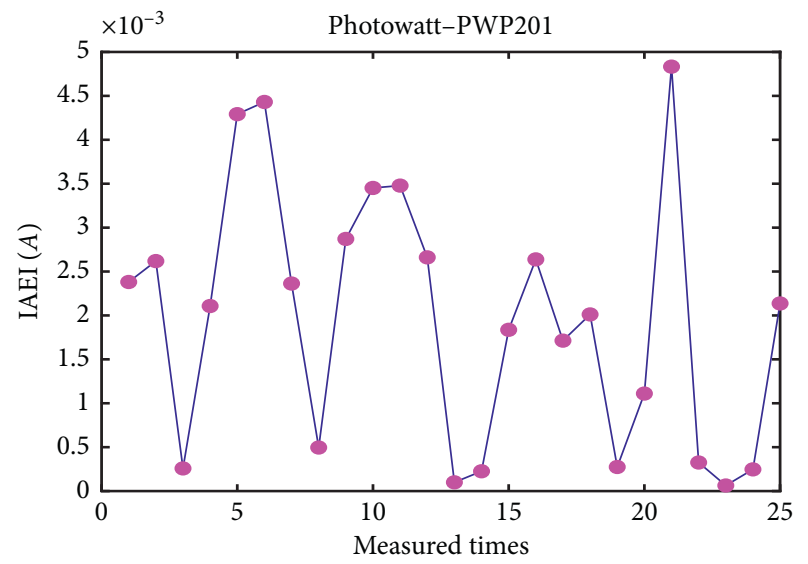

FIgURE 21: The error of IAEI on the photowatt-PWP201 model.

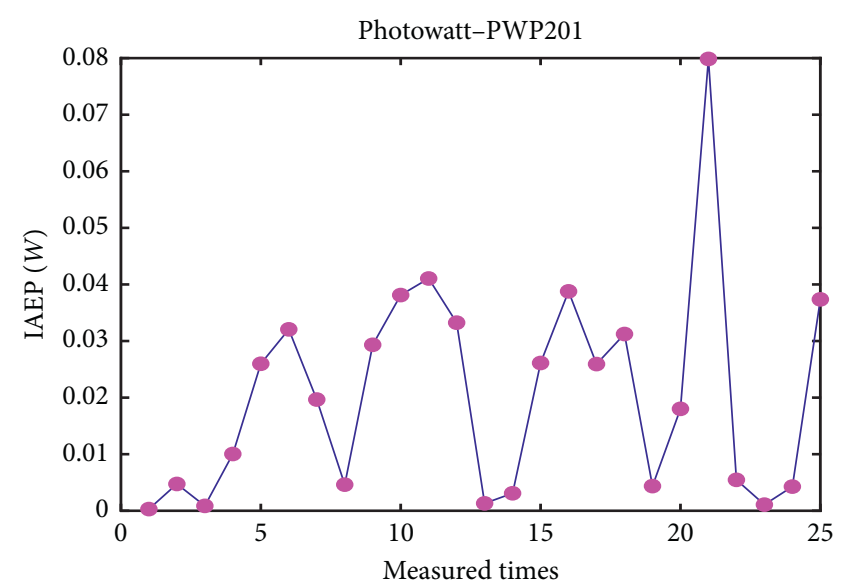

FIGURE 22: The error of IAEP on the photowatt-PWP201 model.

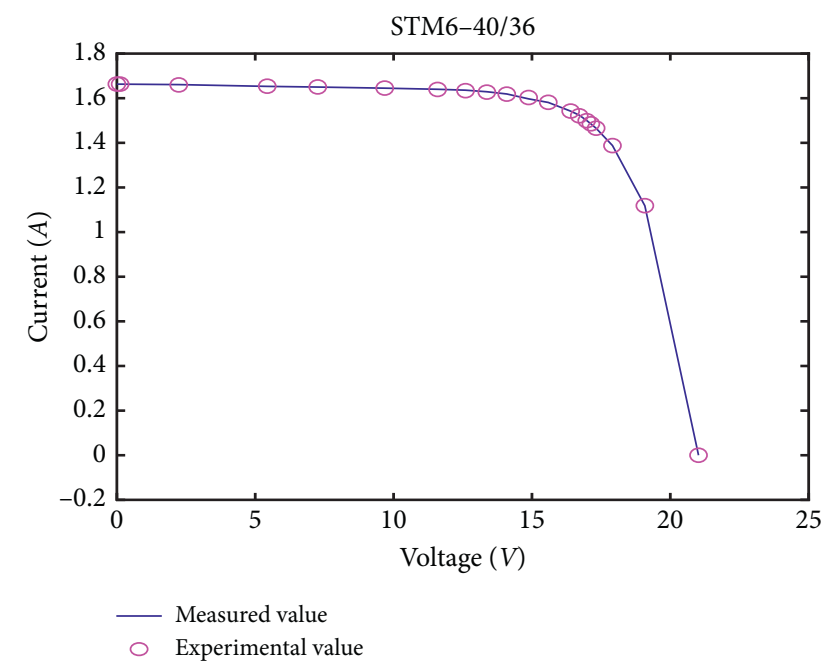

FIgURE 23: The I-V on the STM6-40/36 model.

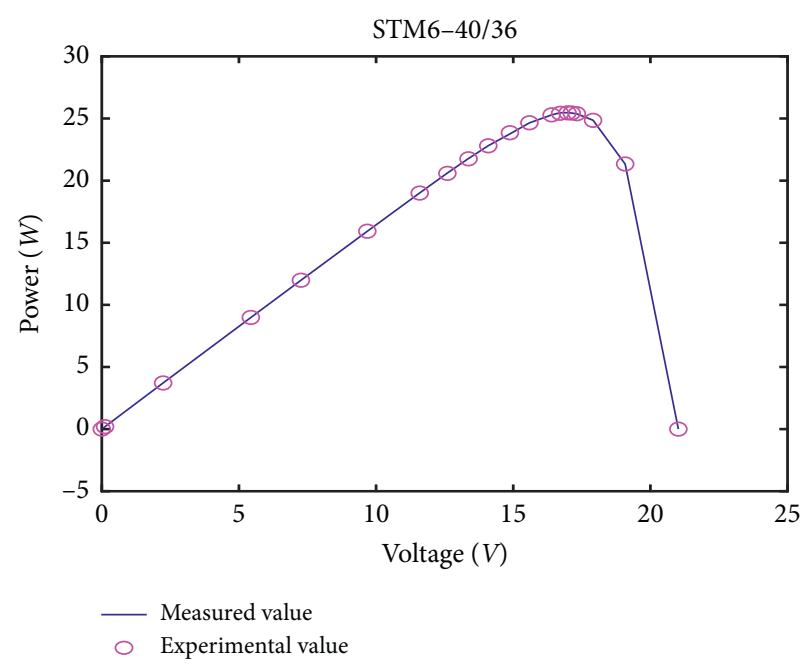

Figure 24: The P-V on the STM6-40/36 model.

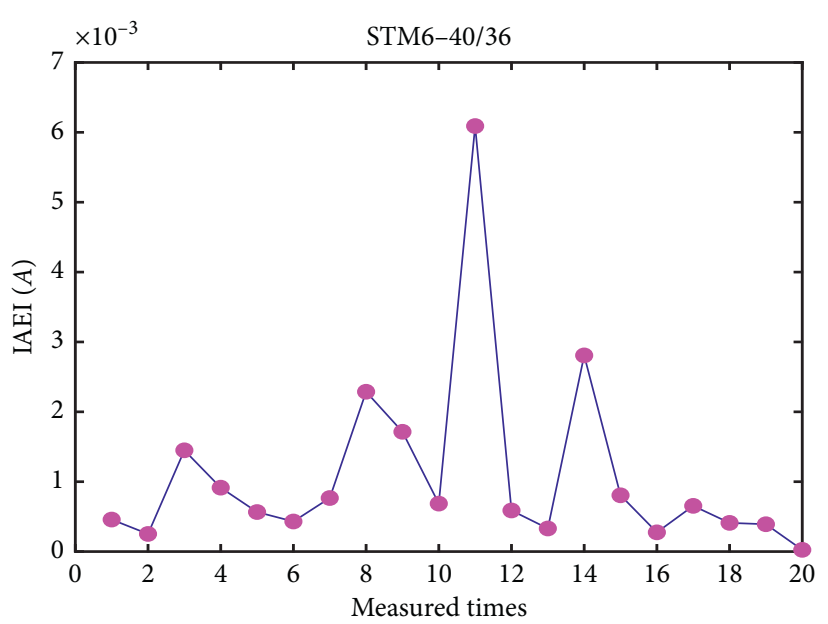

FIgure 25: The error of IAEI on the STM6-40/36 model.

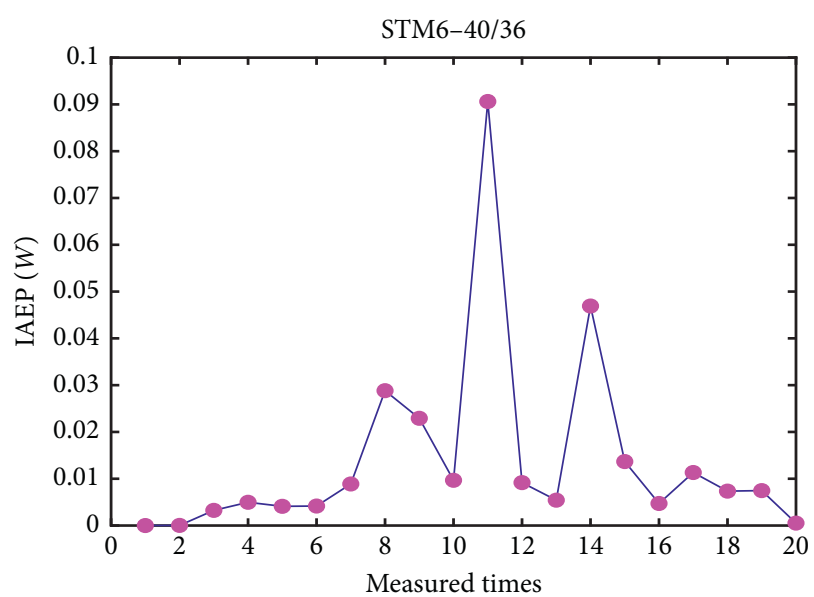

Figure 26: The error of IAEP on the STM6-40/36 model. 


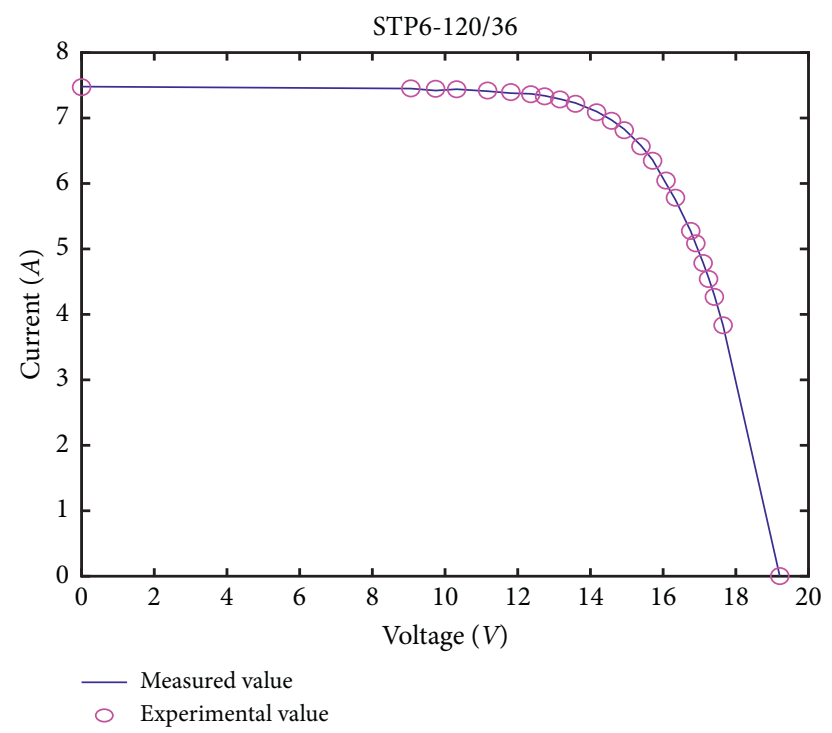

Figure 27: The I-V on the STP6-120/36 model.

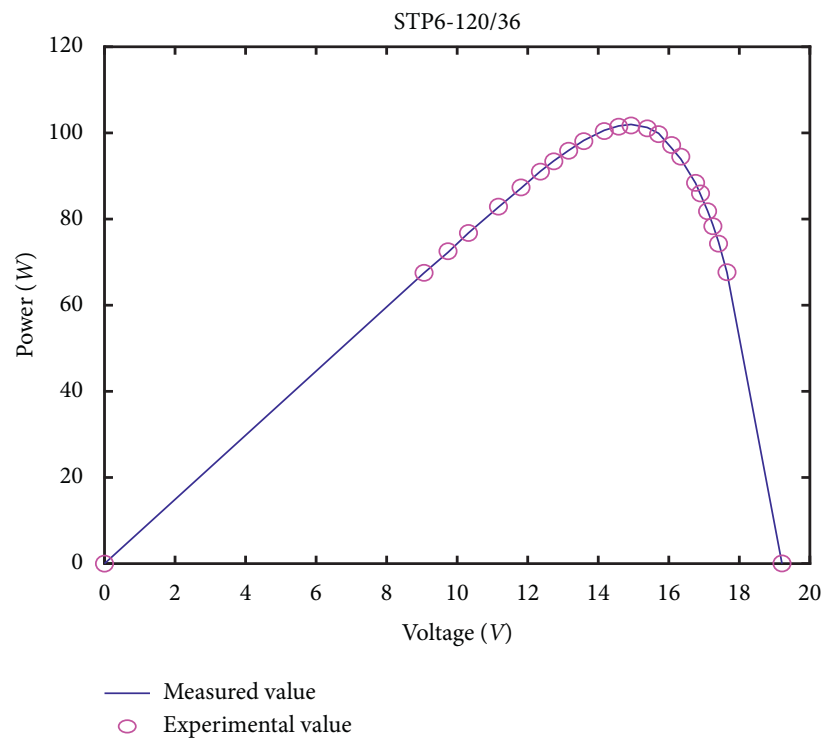

Figure 28: The P-V on the STP6-120/36 model.

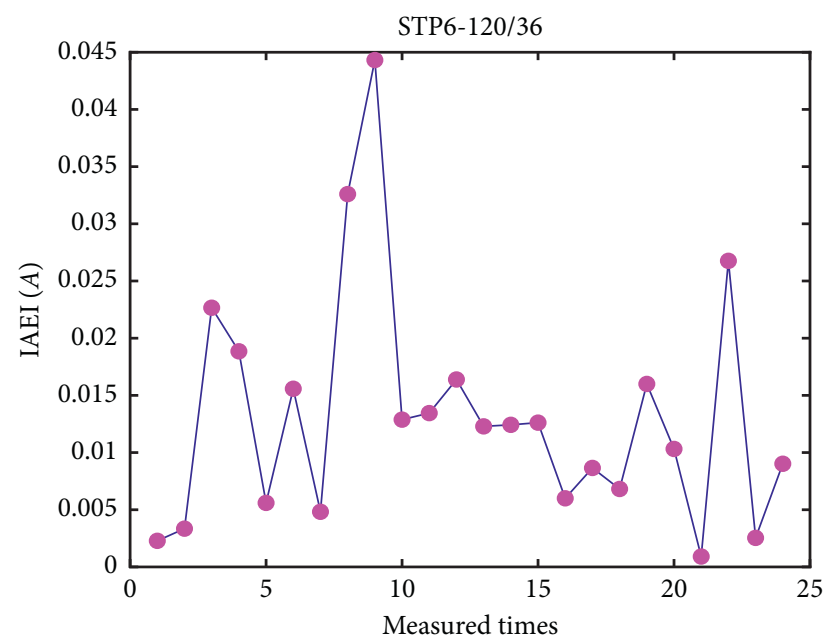

FIgUre 29: The error of IAEI on the STP6-120/36 model. 


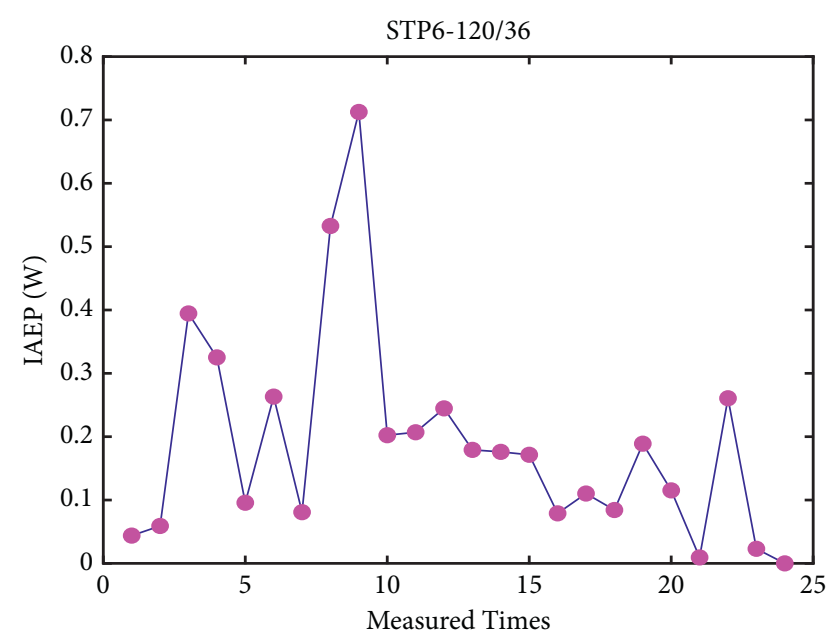

Figure 30: The error of IAEP on the STP6-120/36 model.

parameters to be accurate enough by using EBLSHADE. All the compared results containing the optimal parameters, the BRMSE, WRMSE, MRMSE, SD, and NFE indicate that the EBLSHADE has better capacities of the exploration and exploitation. The reason is that the novel mutation strategies of DE/current-to-or_best/1 and DE/current-to-or_pbest/1 for EBLSHADE can enhance and balance the exploitation capability and exploration capability and enhance the convergence. Rregarding the whole result, the EBLSHADE is considered as a significant method for the parameter optimization of SDM, DDM, and PVM. It is also expected to be applied for optimizing parameters of other PV models.

\section{Conclusion}

In this paper, an enhanced success history adaptive DE(EBLSHADE) has been applied to propose a parameter optimization method for deferent PV models. The effectiveness of the EBLSHADE and parameter optimization method has been verified on estimating parameters of different PV models. The experimental and statistical results with the reliability, accuracy, and computing efficiency show that the EBLSHADE is superior to the other compared algorithms, and the parameter optimization method is an effective method and can design, control, and optimize the PV systems. Therefore, the EBLSHADE is considered as a significant method for optimizing parameters of other PV models.

\section{Data Availability}

The data are available in this paper.

\section{Conflicts of Interest}

The authors declare that they have no conflicts of interest.

\section{Acknowledgments}

This work was supported by the National Natural Science Foundation of China (61771087), the China Education
Ministry Humanities and Social Science Research Youth Fund Project (18YJCZH192), the CERNET Innovation Project (NGII20190605), Yantai Key Research and Development Program (2020YT06000970), Sichuan Science and Technology Program (2019YFG0216 and 2019ZYZF0169), and the $\mathrm{A} \mathrm{Ba}$ Achievements Transformation Program (19CGZH0006).

\section{References}

[1] I. Nassar-eddine, A. Obbadi, Y. Errami, A. El fajri, and M. Agunaou, "Parameter estimation of photovoltaic modules using iterative method and the Lambert W function: a comparative study," Energy Conversion and Management, vol. 119, pp. 37-48, 2016.

[2] U. K. Das, K. S. Tey, M. Seyedmahmoudian et al., "Forecasting of photovoltaic power generation and model optimization: a review," Renewable and Sustainable Energy Reviews, vol. 81, pp. 912-928, 2018.

[3] Y. Liu, Y. Mu, K. Chen, Y. Li, and J. Guo, "Daily activity feature selection in smart homes based on pearson correlation coefficient," Neural Processing Letters, vol. 51, no. 2, pp. 1771-1787, 2020.

[4] T. Li, Z. Qian, and T. He, "Short-term load forecasting with improved CEEMDAN and GWO-based Multiple Kernel ELM," Complexity, vol. 2020, Article ID 1209547, 20 pages, 2020.

[5] Z. Ren, R. Skjetne, A. S. Verma, Z. Jiang, Z. Gao, and K. H. Halse, "Active heave compensation of floating wind turbine installation using a catamaran construction vessel," Marine Structures, vol. 75, Article ID 102868, 2021.

[6] F. Dkhichi, B. Oukarfi, A. Fakkar, and N. Belbounaguia, "Parameter identification of solar cell model using LevenbergMarquardt algorithm combined with simulated annealing," Solar Energy, vol. 110, pp. 781-788, 2014.

[7] W. Deng, H. Zhao, Y. Song, and J. Xu, "An effective improved co-evolution ant colony optimisation algorithm with multistrategies and its application," International Journal of BioInspired Computation, vol. 16, no. 3, pp. 158-170, 2020.

[8] R. Abbassi, A. Abbassi, A. A. Heidari, and S. Mirjalili, "An efficient SALP swarm-inspired algorithm for parameters identification of photovoltaic cell models," Energy Conversion and Management, vol. 179, pp. 362-372, 2019.

[9] T. Li, J. Shi, X. Li, J. Wu, and F. Pan, "Image encryption based on pixel-level diffusion with dynamic filtering and DNA-level permutation with 3D Latin cubes," Entropy, vol. 21, no. 3, p. 319, 2019.

[10] W. Deng, J. Xu, Y. Song, and H. Zhao, "Differential evolution algorithm with wavelet basis function and optimal mutation strategy for complex optimization problem," Applied Soft Computing, Article ID 106724, 2021.

[11] Q. Niu, H. Zhang, and K. Li, "An improved TLBO with elite strategy for parameters identification of PEM fuel cell and solar cell models," International Journal of Hydrogen Energy, vol. 39, no. 8, pp. 3837-3854, 2014.

[12] R. Chen, S.-K. Guo, X.-Z. Wang, and T.-L. Zhang, "Fusion of multi-RSMOTE with fuzzy integral to classify bug reports with an imbalanced distribution," IEEE Transactions on Fuzzy Systems, vol. 27, no. 12, pp. 2406-2420, 2019.

[13] L. L. Jiang, D. L. Maskell, and J. C. Patra, "Parameter estimation of solar cells and modules using an improved adaptive differential evolution algorithm," Applied Energy, vol. 112, pp. 185-193, 2013. 
[14] Y. Liu, X. Wang, Z. Zhai, R. Chen, B. Zhang, and Y. Jiang, "Timely daily activity recognition from headmost sensor events," ISA Transactions, vol. 94, pp. 379-390, 2019.

[15] S. Li, Q. Gu, W. Gong, and B. Ning, "An enhanced adaptive differential evolution algorithm for parameter extraction of photovoltaic models," Energy Conversion and Management, vol. 205, Article ID 112443, 2020.

[16] W. Deng, J. Xu, X.-Z. Gao, and H. Zhao, “An enhanced MSIQDE algorithm with novel multiple strategies for global optimization problems," IEEE Transactions on Systems, Man, and Cybernetics: Systems, p. 1, 2020.

[17] D. H. Muhsen, A. B. Ghazali, T. Khatib, and I. A. Abed, "A comparative study of evolutionary algorithms and adapting control parameters for estimating the parameters of a singlediode photovoltaic module's model," Renewable Energy, vol. 96, pp. 377-389, 2016.

[18] W. Deng, H. Liu, J. Xu, H. Zhao, and Y. Song, "An improved quantum-inspired differential evolution algorithm for deep belief network," IEEE Transactions on Instrumentation and Measurement, vol. 69, no. 10, pp. 7319-7327, 2020.

[19] H. L. Chen, S. M. Li, A. A. Heidari et al., "Efficient multipopulation outpost fruit fly-driven optimizers: framework and advances in support vector machines," Expert Systems with Applications, vol. 142, Article ID 112999, 2020.

[20] W. Deng, J. Xu, H. Zhao, and Y. Song, "A novel gate resource allocation method using improved PSO-based QEA," IEEE Transactions on Intelligent Transportation Systems, vol. 14, 2020.

[21] A. W. Mohamed, A. A. Hadi, and A. K. Mohamed, "Gainingsharing knowledge based algorithm for solving optimization problems: a novel nature-inspired algorithm," International Journal of Machine Learning and Cybernetics, vol. 11, no. 7, pp. 1501-1529, 2020.

[22] M. Zagrouba, A. Sellami, M. Bouaïcha, and M. Ksouri, "Identification of PV solar cells and modules parameters using the genetic algorithms: application to maximum power extraction," Solar Energy, vol. 84, no. 5, pp. 860-866, 2010.

[23] K. Ishaque, Z. Salam, S. Mekhilef, and A. Shamsudin, "Parameter extraction of solar photovoltaic modules using penalty-based differential evolution," Applied Energy, vol. 99, pp. 297-308, 2012.

[24] M. Merchaoui, A. Sakly, and M. F. Mimouni, "Particle swarm optimisation with adaptive mutation strategy for photovoltaic solar cell/module parameter extraction," Energy Conversion and Management, vol. 175, pp. 151-163, 2018.

[25] K. Yu, B. Qu, C. Yue, S. Ge, X. Chen, and J. Liang, “A performance-guided JAYA algorithm for parameters identification of photovoltaic cell and module," Applied Energy, vol. 237, pp. 241-257, 2019.

[26] X. Chen, B. Xu, C. Mei, Y. Ding, and K. Li, "Teachinglearning-based artificial bee colony for solar photovoltaic parameter estimation," Applied Energy, vol. 212, pp. 1578$1588,2018$.

[27] S. Li, W. Gong, X. Yan et al., "Parameter extraction of photovoltaic models using an improved teaching-learningbased optimization," Energy Conversion and Management, vol. 186, pp. 293-305, 2019.

[28] D. Oliva, E. Cuevas, and G. Pajares, "Parameter identification of solar cells using artificial bee colony optimization," Energy, vol. 72, no. 7, pp. 93-102, 2014.

[29] Z. Wu, D. Yu, and X. Kang, "Parameter identification of photovoltaic cell model based on improved ant lion optimizer," Energy Conversion and Management, vol. 151, pp. 107-115, 2017.
[30] J. P. Ram, T. S. Babu, T. Dragicevic, and N. Rajasekar, “A new hybrid bee pollinator flower pollination algorithm for solar PV parameter estimation," Energy Conversion and Management, vol. 135, pp. 463-476, 2017.

[31] D. Oliva, M. Abd El Aziz, and A. Ella Hassanien, "Parameter estimation of photovoltaic cells using an improved chaotic whale optimization algorithm," Applied Energy, vol. 200, pp. 141-154, 2017.

[32] S. Xu and Y. Wang, "Parameter estimation of photovoltaic modules using a hybrid flower pollination algorithm," Energy Conversion and Management, vol. 144, pp. 53-68, 2017.

[33] H. Chen, S. Jiao, M. Wang, A. A. Heidari, and X. Zhao, "Parameters identification of photovoltaic cells and modules using diversification-enriched Harris hawks optimization with chaotic drifts," Journal of Cleaner Production, vol. 244, Article ID 118778, 2020.

[34] W. Long, S. Cai, J. Jiao, M. Xu, and T. Wu, "A new hybrid algorithm based on grey wolf optimizer and cuckoo search for parameter extraction of solar photovoltaic models," Energy Conversion and Management, vol. 203, Article ID 112243, 2020.

[35] H. Chen, S. Jiao, A. A. Heidari, M. Wang, X. Chen, and X. Zhao, "An opposition-based sine cosine approach with local search for parameter estimation of photovoltaic models," Energy Conversion and Management, vol. 195, pp. 927-942, 2019.

[36] S. Cai, G. Bao, X. Ma et al., "Parameters optimization of the dust absorbing structure for photovoltaic panel cleaning robot based on orthogonal experiment method," Journal of Cleaner Production, vol. 217, pp. 724-731, 2019.

[37] R. Storn and K. Price, "Differential evolution a simple and efficient heuristic for global optimization over continuous spaces," Journal of Global Optimization, vol. 11, no. 4, pp. 341-359, 1997.

[38] Y.-P. Chang, "An ant direction hybrid differential evolution algorithm in determining the tilt angle for photovoltaic modules," Expert Systems with Applications, vol. 37, no. 7, pp. 5415-5422, 2010.

[39] W. Gong and Z. Cai, "Parameter extraction of solar cell models using repaired adaptive differential evolution," Solar Energy, vol. 94, pp. 209-220, 2013.

[40] D. H. Muhsen, A. B. Ghazali, T. Khatib, and I. A. Abed, "Extraction of photovoltaic module model's parameters using an improved hybrid differential evolution/electromagnetismlike algorithm," Solar Energy, vol. 119, pp. 286-297, 2015.

[41] C. Chellaswamy and R. Ramesh, "Parameter extraction of solar cell models based on adaptive differential evolution algorithm," Renewable Energy, vol. 97, pp. 823-837, 2016.

[42] A. W. Mohamed and S. A. Abdulaziz, "Differential evolution with novel mutation and adaptive crossover strategies for solving large-scale global optimization problems," Applied Computational Intelligence and Soft Computing, vol. 2017, Article ID 7974218, 18 pages, 2017.

[43] H. Zhang, D. Yue, X. Xie, C. Dou, and F. Sun, "Gradient decent based multi-objective cultural differential evolution for short-term hydrothermal optimal scheduling of economic emission with integrating wind power and photovoltaic power," Energy, vol. 122, pp. 748-766, 2017.

[44] A. W. Mohamed, "Solving large-scale global optimization problems using enhanced adaptive differential evolution algorithm," Complex \& Intelligent Systems, vol. 3, no. 4, pp. 205-231, 2017.

[45] H. Rashidi and J. Khorshidi, "Exergoeconomic analysis and optimization of a solar based multigeneration system using 
multiobjective differential evolution algorithm," Journal of Cleaner Production, vol. 170, pp. 978-990, 2018.

[46] A. W. Mohamed, "Solving stochastic programming problems using new approach to differential evolution algorithm," Egyptian Informatics Journal, vol. 18, no. 2, pp. 75-86, 2017.

[47] M. A. M. Ramli, H. R. E. H. Bouchekara, and A. S. Alghamdi, "Optimal sizing of PV/wind/diesel hybrid microgrid system using multi-objective self-adaptive differential evolution algorithm," Renewable Energy, vol. 121, pp. 400-411, 2018.

[48] A. W. Mohamed and P. N. Suganthan, "Real-parameter unconstrained optimization based on enhanced fitnessadaptive differential evolution algorithm with novel mutation," Soft Computing, vol. 22, no. 10, pp. 3215-3235, 2018.

[49] A. W. Mohamed and A. K. Mohamed, "Adaptive guided differential evolution algorithm with novel mutation for numerical optimization," International Journal of Machine Learning and Cybernetics, vol. 10, no. 2, pp. 253-277, 2019.

[50] K. S. Tey, S. Mekhilef, M. Seyedmahmoudian, B. Horan, A. T. Oo, and A. Stojcevski, "Improved differential evolutionbased MPPT algorithm using SEPIC for PV systems under partial shading conditions and load variation," IEEE Transactions on Industrial Informatics, vol. 14, no. 10, pp. 43224333, 2018.

[51] G. Xiong, J. Zhang, X. Yuan, D. Shi, Y. He, and G. Yao, "Parameter extraction of solar photovoltaic models by means of a hybrid differential evolution with whale optimization algorithm," Solar Energy, vol. 176, pp. 742-761, 2018.

[52] A. A. Hadi, A. W. Mohamed, and K. M. Jambi, "LSHADESPA memetic framework for solving large-scale optimization problems," Complex \& Intelligent Systems, vol. 5, no. 1, pp. 25-40, 2019.

[53] A. K. Mohamed and A. W. Mohamed, "Real-parameter unconstrained optimization based on enhanced agde algorithm," in Machine Learning Paradigms: Theory and Application. Studies in Computational Intelligence, A. Hassanien, Ed., p. 801, Springer, Berlin, Germany, 2019.

[54] S. Li, W. Gong, X. Yan, C. Hu, D. Bai, and L. Wang, "Parameter estimation of photovoltaic models with memetic adaptive differential evolution," Solar Energy, vol. 190, pp. 465-474, 2019.

[55] A. W. Mohamed, A. K. Mohamed, E. Z. Elfeky, and M. Saleh, "Enhanced directed differential evolution algorithm for solving constrained engineering optimization problems," International Journal of Applied Metaheuristic Computing, vol. 10, no. 1, pp. 1-28, 2019.

[56] I. O. Essiet, Y. Sun, and Z. Wang, "Optimized energy consumption model for smart home using improved differential evolution algorithm," Energy, vol. 172, pp. 354-365, 2019.

[57] A. W. Mohamed, A. A. Hadi, and K. M. Jambi, "Novel mutation strategy for enhancing SHADE and LSHADE algorithms for global numerical optimization," Swarm and Evolutionary Computation, vol. 50, Article ID 100455, 2019.

[58] T. Easwarakhanthan, J. Bottin, I. Bouhouch, and C. Boutrit, "Nonlinear minimization algorithm for determining the solar cell parameters with microcomputers," International Journal of Solar Energy, vol. 4, no. 1, pp. 1-12, 1986.

[59] N. T. Tong and W. Pora, "A parameter extraction technique exploiting intrinsic properties of solar cells," Applied Energy, vol. 176, pp. 104-115, 2016.

[60] Y. Song, D. Wu, W. Deng et al., "MPPCEDE: multi-population parallel co-evolutionary differential evolution for parameter optimization," Energy Conversion and Management, vol. 228, Article ID 113661, 2021.
[61] K. Yu, J. J. Liang, B. Y. Qu, X. Chen, and H. Wang, "Parameters identification of photovoltaic models using an improved JAYA optimization algorithm," Energy Conversion and Management, vol. 150, pp. 742-753, 2017.

[62] J. J. Liang, A. K. Qin, P. N. Suganthan, and S. Baskar, "Comprehensive learning particle swarm optimizer for global optimization of multimodal functions," IEEE Transactions on Evolutionary Computation, vol. 10, no. 3, pp. 281-295, 2006.

[63] F. Zou, L. Wang, X. Hei, and D. Chen, "Teaching-learningbased optimization with learning experience of other learners and its application," Applied Soft Computing, vol. 37, pp. 725-736, 2015.

[64] K. Yu, X. Chen, X. Wang, and Z. Wang, "Parameters identification of photovoltaic models using self-adaptive teachinglearning-based optimization," Energy Conversion and Management, vol. 145, pp. 233-246, 2017.

[65] W. Gong, Z. Cai, and C. X. Ling, "DE/BBO: a hybrid differential evolution with biogeography-based optimization for global numerical optimization," Soft Computing, vol. 15, no. 4, pp. 645-665, 2010.

[66] D. Chen, F. Zou, R. Lu, and P. Wang, "Learning backtracking search optimisation algorithm and its application," Information Sciences, vol. 376, pp. 71-94, 2017.

[67] X. Chen, H. Tianfield, C. Mei, W. Du, and G. . Liu, "Biogeography-based learning particle swarm optimization," Soft Computing, vol. 21, pp. 1-23, 2016.

[68] X. Chen, H. Tianfield, W. Du, and G. Liu, "Biogeographybased optimization with covariance matrix based migration," Applied Soft Computing, vol. 45, pp. 71-85, 2016.

[69] K. Yu, J. J. Liang, B. Y. Qu, Z. Cheng, and H. Wang, "Multiple learning backtracking search algorithm for estimating parameters of photovoltaic models," Applied Energy, vol. 226, pp. $408-422,2018$. 